\title{
A deletion in the STA1 promoter determines maltotriose and starch utilization in STA1+ Saccharomyces cerevisiae strains
}

\author{
Kristoffer Krogerus $^{1,2}$ (D) $\cdot$ Frederico Magalhães ${ }^{1} \cdot$ Joosu Kuivanen ${ }^{1,3} \cdot$ Brian Gibson $^{1}$
}

Received: 3 June 2019 / Revised: 6 July 2019 / Accepted: 9 July 2019 / Published online: 26 July 2019

(C) The Author(s) 2019

\begin{abstract}
Diastatic strains of Saccharomyces cerevisiae are common contaminants in beer fermentations and are capable of producing an extracellular STA1-encoded glucoamylase. Recent studies have revealed variable diastatic ability in strains tested positive for STA1, and here, we elucidate genetic determinants behind this variation. We show that poorly diastatic strains have a 1162-bp deletion in the promoter of STA1. With CRISPR/Cas9-aided reverse engineering, we show that this deletion greatly decreases the ability to grow in beer and consume dextrin, and the expression of STA1. New PCR primers were designed for differentiation of highly and poorly diastatic strains based on the presence of the deletion in the STA1 promoter. In addition, using publically available whole genome sequence data, we show that the STA1 gene is prevalent among the 'Beer 2'/'Mosaic Beer' brewing strains. These strains utilize maltotriose efficiently, but the mechanisms for this have been unknown. By deleting STA1 from a number of highly diastatic strains, we show here that extracellular hydrolysis of maltotriose through STA1 appears to be the dominant mechanism enabling maltotriose use during wort fermentation in STA1+ strains. The formation and retention of STA1 seems to be an alternative evolutionary strategy for efficient utilization of sugars present in brewer's wort. The results of this study allow for the improved reliability of molecular detection methods for diastatic contaminants in beer and can be exploited for strain development where maltotriose use is desired.
\end{abstract}

Keywords Yeast $\cdot$ Beer $\cdot$ Dextrin $\cdot$ Starch $\cdot$ Diastatic $\cdot$ Genome

\section{Introduction}

Diastatic strains of Saccharomyces cerevisiae are considered spoilage microorganisms in industrial beer fermentations (Andrews and Gilliland 1952; Meier-Dörnberg et al. 2018). These strains, formerly known as

Electronic supplementary material The online version of this article (https://doi.org/10.1007/s00253-019-10021-y) contains supplementary material, which is available to authorized users.

Kristoffer Krogerus

kristoffer.krogerus@gmail.com

1 VTT Technical Research Centre of Finland, Tietotie 2, P.O. Box 1000, FI-02044 VTT Espoo, Finland

2 Department of Biotechnology and Chemical Technology, Aalto University, School of Chemical Technology, Kemistintie 1, Aalto, P.O. Box 16100, FI-00076 Espoo, Finland

3 Tampere University, Tampere, Finland
Saccharomyces diastaticus, are capable of producing an extracellular glucoamylase that enables fermentation of starch and oligosaccharides (Andrews and Gilliland 1952; Erratt and Stewart 1981; Yamashita et al. 1984; Kleinman et al. 1988). This, in turn, causes superattenuation in the beer, which leads to increased carbon dioxide and ethanol levels, as well as the possibility of off-flavours and a drier mouthfeel (Meier-Dörnberg et al. 2018). In the case of contamination in packaged beer, diastatic $S$. cerevisiae may even cause exploding bottles and cans, endangering the consumer.

The extracellular glucoamylase in diastatic $S$. cerevisiae is coded for by the STA genes (Tamaki 1978; Yamashita et al. 1985a, b). Three highly homologous STA genes (STA1-3) have been described (Tamaki 1978; Lambrechts et al. 1991). In addition, DEX1-2 has been used to describe genes encoding extracellular glucoamylases (Erratt and Stewart 1981); however, these were later shown to be allelic to the STA genes (Erratt and Nasim 1986). The STAl gene appears to be 
chimeric, consisting of rearranged gene fragments from both FLOI1 and SGA1 (Yamashita et al. 1987; Lo and Dranginis 1996). The $5^{\prime}$ end of STA1 is homologous to FLO11, a gene encoding a membrane-bound flocculin promoting flocculation, while the $3^{\prime}$ end is homologous to $S G A 1$, a gene encoding an intracellular glucoamylase used during sporulation. The catalytic domain is located in the SGA1-derived peptide, while the FLO11-derived peptide allows for extracellular secretion of the protein (Adam et al. 2004). The upstream sequences of STA1 and FLO11 are also nearly identical, meaning that these genes are largely co-regulated (Gagiano et al. 1999).

Current detection methods for diastatic $S$. cerevisiae rely mainly on either the microbiological detection through culturing on specialized selective growth media, or the molecular detection of the STA1 gene through conventional or quantitative PCR (Yamauchi et al. 1998; van der Aa 1998; Brandl 2006; Meier-Dörnberg et al. 2018). The main weakness of the microbiological methods is that they are time-consuming. While the molecular methods are rapid, a recent study (Meier-Dörnberg et al. 2018) has revealed that there is considerable variability in diastatic ability and beer-spoilage potential in strains carrying the STA1 gene. In fact, some strains that carry the STA 1 gene do not show spoilage potential, and these benign strains would be erroneously flagged as diastatic with the current molecular methods.

In this study, we examined the diastatic ability of a range of STA1+S. cerevisiae strains. We also sequenced the open reading frame and upstream sequence of STA1 in these same strains to search for polymorphisms that might explain the variable diastatic ability. Sequencing revealed that the poorly diastatic strains have a common 1162-bp deletion in the promoter of STA1. Through CRISPR/Cas9-aided reverse engineering, we show that this deletion greatly decreases diastatic ability and the expression of STA1. We designed new PCR primers targeting this deleted region, and these can be used to differentiate highly and poorly diastatic strains.

In addition, using publically available whole genome sequence data, we show that the STA1 gene is prevalent in both the 'Beer 2' ('Mosaic Beer') population and, surprisingly, the 'French Guaina, human isolate' population (Gallone et al. 2016; Peter et al. 2018). Strains in the 'Beer 2' population have been shown to utilize maltotriose efficiently, despite carrying frameshift mutations in AGT1/ MAL11 (Gallone et al. 2016). These strains are therefore likely to utilize alternative mechanisms for maltotriose use. By deleting STA 1 from a number of highly diastatic strains, we show here that STA1 appears to have a central role in enabling maltotriose use in these strains during wort fermentation.

\section{Materials and Methods}

\section{Yeast strains}

A list of strains used in this study can be found in Table 1.

\section{Dextrin fermentation}

The ability to ferment dextrin was tested in minimal growth media with dextrin as the sole carbon source. Strains were grown overnight in YP-Glucose (2\%), after which $20 \mu \mathrm{L}$ of overnight culture was used to inoculate $2 \mathrm{~mL}$ microcentrifuge tubes containing $1 \mathrm{~mL}$ of dextrin media $(0.67 \%$ YNB without amino acids, $1 \%$ dextrin from potato starch). The tubes were incubated at room temperature for 3 weeks. Samples were drawn each week and analysed with HPLC to determine the amount of dextrin consumed.

\section{Growth on starch agar}

The ability to grow on solid media containing starch as the sole carbon source was tested on agar plates (Meier-Dörnberg et al. 2018). Agar plates were prepared containing $0.67 \%$ YNB/wo amino acids, $1.5 \%$ soluble starch (Merck, Darmstadt, Germany), and $40 \mathrm{mg} / \mathrm{L}$ bromophenol blue. $\mathrm{pH}$ was adjusted to 5.2 with $0.1 \mathrm{M} \mathrm{HCl}$. Yeast strains were grown overnight in YP-Glucose, washed twice with sterile MQ- $\mathrm{H}_{2} \mathrm{O}$ and then resuspended to an OD600 of 1 . Aliquots of yeast suspension $(100 \mu \mathrm{L})$ were spread out on agar plates. The plates were incubated in an anaerobic jar (Anoxomat AN2CTS, Mart Microbiology, Drachten, Netherlands) at 25 ${ }^{\circ} \mathrm{C}$ for 4 weeks.

\section{Growth in beer}

The spoilage potential of STA $1+$ strains was assessed by growing them in beer. Beer was produced by inoculating $1 \mathrm{~L}$ of 10 ${ }^{\circ}$ Plato all-malt wort with Saccharomyces pastorianus A15 and fermenting at $25^{\circ} \mathrm{C}$ for 1 week. The beer was centrifuged and sterile-filtered $(0.45 \mu \mathrm{m}$, followed by $0.22 \mu \mathrm{m})$. The beer was analysed with high-performance liquid chromatography (HPLC) and determined to contain less than $1 \mathrm{~g} / \mathrm{L}$ each of maltose and maltotriose. Yeast strains were grown overnight in YP-Glucose, washed twice with sterile deionized water (MQ- $\mathrm{H}_{2} \mathrm{O}$ ), and then resuspended to an OD600 of 1. Sterilefiltered beer $(4750 \mu \mathrm{L})$ was inoculated with $250 \mu \mathrm{L}$ of preculture for a starting OD600 of 0.05 . The beer cultivations were incubated statically in an anaerobic jar (Anoxomat AN2CTS, Mart Microbiology, Drachten, Netherlands) at 25 ${ }^{\circ} \mathrm{C}$ for 3 weeks. The optical density was monitored once a 
Table 1 Yeast strains used in the study

\begin{tabular}{|c|c|c|c|c|c|}
\hline Code & Alternative name & Species & STA1 & $\begin{array}{l}\text { 1162-bp deletion in STAI } \\
\text { promoter }\end{array}$ & Source \\
\hline A15 & VTT-A83015 & S. pastorianus & - & NA & $\begin{array}{l}\text { VTT Culture Collection, Espoo, } \\
\text { Finland }\end{array}$ \\
\hline A60 & VTT-A75060 & S. cerevisiae & - & NA & $\begin{array}{l}\text { VTT Culture Collection, Espoo, } \\
\text { Finland }\end{array}$ \\
\hline WLP007 & & S. cerevisiae & - & NA & White Labs Inc., San Diego, CA, USA \\
\hline WLP023 & & S. cerevisiae & - & NA & White Labs Inc., San Diego, CA, USA \\
\hline TUM PI BA 109 & & S. cerevisiae & + & - & $\begin{array}{l}\text { BLQ Weihenstephan, Freising, } \\
\text { Germany }\end{array}$ \\
\hline TUM 3-D-2 & & S. cerevisiae & + & - & $\begin{array}{l}\text { BLQ Weihenstephan, Freising, } \\
\text { Germany }\end{array}$ \\
\hline TUM PI BA 45 & & S. cerevisiae & + & + & $\begin{array}{l}\text { BLQ Weihenstephan, Freising, } \\
\text { Germany }\end{array}$ \\
\hline TUM PI BB 105 & & S. cerevisiae & + & + & $\begin{array}{l}\text { BLQ Weihenstephan, Freising, } \\
\text { Germany }\end{array}$ \\
\hline TUM PI BA 31 & & S. cerevisiae & + & + & $\begin{array}{l}\text { BLQ Weihenstephan, Freising, } \\
\text { Germany }\end{array}$ \\
\hline TUM 71 & & S. cerevisiae & + & - & $\begin{array}{l}\text { BLQ Weihenstephan, Freising, } \\
\text { Germany }\end{array}$ \\
\hline TUM 3-H-2 & & S. cerevisiae & + & - & $\begin{array}{l}\text { BLQ Weihenstephan, Freising, } \\
\text { Germany }\end{array}$ \\
\hline WY3711 & & S. cerevisiae & + & - & Wyeast Laboratories, Odell, OR, USA \\
\hline WLP565 & & S. cerevisiae & + & + & White Labs Inc., San Diego, CA, USA \\
\hline WLP566 & & S. cerevisiae & + & + & White Labs Inc., San Diego, CA, USA \\
\hline WLP570 & & S. cerevisiae & + & + & White Labs Inc., San Diego, CA, USA \\
\hline WLP644 & & S. cerevisiae & + & + & White Labs Inc., San Diego, CA, USA \\
\hline Ontario Farmhouse & & S. cerevisiae & + & + & $\begin{array}{l}\text { Escarpment Laboratories, Guelph, } \\
\text { Canada }\end{array}$ \\
\hline Wild Thing & & S. cerevisiae & + & + & $\begin{array}{l}\text { Escarpment Laboratories, Guelph, } \\
\text { Canada }\end{array}$ \\
\hline A62 & VTT-A81062 & S. cerevisiae & + & + & $\begin{array}{l}\text { VTT Culture Collection, Espoo, } \\
\text { Finland }\end{array}$ \\
\hline \multicolumn{6}{|c|}{ CRISPR/Cas9-modified strains } \\
\hline WY3711_D1 & $\begin{array}{l}\mathrm{P}_{\text {stal }}-\Delta 1 \\
\quad(\text { STA1:c.-1370_-290del) }\end{array}$ & S. cerevisiae & + & + & This study \\
\hline WY3711_S1 & $\begin{array}{l}\text { sta1- } \Delta 0(\text { STA1:c.-1370_+ } \\
\quad 2421 \mathrm{del})\end{array}$ & S. cerevisiae & - & NA & This study \\
\hline $\begin{array}{l}\text { TUM PI BA } 109 \\
\text { S1 }\end{array}$ & stal- $\Delta 0$ (as above) & S. cerevisiae & - & NA & This study \\
\hline TUM 71_S1 & stal- $\Delta 0$ (as above) & S. cerevisiae & - & NA & This study \\
\hline
\end{tabular}

week, and the final amount of dextrin consumed was analysed with HPLC after 3 weeks.

\section{Wort fermentation and analysis}

$80 \mathrm{~mL}$-scale fermentations were carried out in $100 \mathrm{~mL}$ Schott bottles capped with glycerol-filled airlocks. Yeast strains were grown overnight in $25 \mathrm{~mL}$ YP-Maltose $(4 \%)$ at $25^{\circ} \mathrm{C}$. The pre-cultured yeast was then inoculated into $80 \mathrm{~mL}$ of $15^{\circ} \mathrm{P}$ allmalt wort at a rate of $1 \mathrm{~g}$ fresh yeast $\mathrm{L}^{-1}$. Fermentations were carried out in triplicate at $25^{\circ} \mathrm{C}$ for 15 days. Fermentations were monitored by mass lost as $\mathrm{CO}_{2}$. Samples were also drawn throughout fermentation and analysed with HPLC to monitor sugar consumption.

Concentrations of fermentable sugars and dextrin were measured by HPLC using a Waters 2695 Separation Module 
and Waters System Interphase Module liquid chromatograph coupled with a Waters 2414 differential refractometer (Waters Co., Milford, MA, USA). An Aminex HPX-87H organic acid analysis column $(300 \times 7.8 \mathrm{~mm}$, Bio-Rad $)$ was equilibrated with $5 \mathrm{mM} \mathrm{H}_{2} \mathrm{SO}_{4}$ (Titrisol, Merck, Darmstadt, Germany) in water at $55^{\circ} \mathrm{C}$ and samples were eluted with $5 \mathrm{mM} \mathrm{H}_{2} \mathrm{SO}_{4}$ in water at a $0.3 \mathrm{~mL} / \mathrm{min}$ flow rate.

\section{Maltotriose uptake assay}

The zero-trans maltotriose uptake rate was assayed using [U- $\left.{ }^{14} \mathrm{C}\right]-$ maltotriose as described by Lucero et al. (1997). Yeast strains were grown in YP-Maltose at $20{ }^{\circ} \mathrm{C}$ to an OD600 between 4 and 8 prior to uptake measurement. Yeast was harvested by centrifugation, washed with ice-cold water, and then with icecold $0.1 \mathrm{M}$ tartrate-Tris ( $\mathrm{pH} 4.2$ ). and finally resuspended in the same buffer at a concentration of $200 \mathrm{mg}$ of fresh yeast $\mathrm{mL}^{-1}$. The uptake rate was determined at $20{ }^{\circ} \mathrm{C}$ using $5 \mathrm{mM}$ of $\left[\mathrm{U}-{ }^{14} \mathrm{C}\right]$-maltotriose (ARC 627, American Radiolabeled Chemicals Inc., St. Louis, MO, USA) in 0.1 M tartrate-Tris ( $\mathrm{pH} 4.2)$ with $1 \mathrm{~min}$ incubation time. $\left[\mathrm{U}^{14} \mathrm{C}\right]$-maltotriose was repurified before use as described by Dietvorst et al. (2005).

\section{Sequencing of STA1}

The STA 1 open reading frame and promoter (2.5 kb upstream) were amplified by PCR and then sequenced using Sanger sequencing. A nested PCR approach was used to prevent PCR primers from amplifying fragments from FLO11 and $S G A 1$. First, a $5.2 \mathrm{~kb}$ amplicon was produced using primers STA1_Full_Fw and STA1_Full_Rv (Table 2). The amplicon was diluted 1:500 in MQ- $\mathrm{H}_{2} \mathrm{O}$ and used as template DNA for the ten PCR reactions described in Table 2. All PCR reactions were carried out with Phusion High-Fidelity PCR Master Mix with HF Buffer (Thermo Scientific, Vantaa, Finland) and primer concentrations of $0.5 \mu \mathrm{M}$. Amplicons were cleaned using the QIAquick PCR purification kit of Qiagen (Hilden, Germany) and sequenced at Seqlab-Microsynth (Goettingen, Germany). Sequences were aligned in Geneious 10.0.9 (Biomatters, Auckland, New Zealand). The sequences are available in Supplementary Data 1.

\section{Multiplex PCR to detect presence of deletion in STA1 promoter}

The presence of the STAl gene was tested with PCR using the previously published primers SD-5A and SD-6B (Yamauchi et al. 1998). These primers amplify an 868-bp fragment from the STA 1 gene. In addition, a new primer pair (STA1_UAS_Fw and STA1_UAS_Rv; Table 2) was designed to detect whether the STA1+ yeast strains had the full promoter sequence. Both primers bind within the 1162-bp region that was found to be deleted in the poorly diastatic strains, and they amplify a 599-bp fragment. Primers were tested in separate and multiplex PCR reactions. PCR reactions were carried out with Phusion High-Fidelity PCR Master Mix with HF Buffer (Thermo Scientific, Vantaa, Finland) and primer concentrations of $0.5 \mu \mathrm{M}$. The following PCR programme was used: $98^{\circ} \mathrm{C} 30 \mathrm{sec},\left(98^{\circ} \mathrm{C} 10 \mathrm{sec}, 63^{\circ} \mathrm{C} 15 \mathrm{sec}, 72{ }^{\circ} \mathrm{C} 30 \mathrm{sec}\right) \times 30$ cycles, $72{ }^{\circ} \mathrm{C} 10 \mathrm{~min}$. PCR products were separated and visualized on $1.0 \%$ agarose gels.

To test the primers in a simulated real-world scenario, we performed PCR on DNA extracted from cultures of lager yeast contaminated with various ratios of diastatic $S$. cerevisiae. Cultures of S. cerevisiae WY3711 and S. pastorianus A15 were grown overnight in YP-Glucose, and duplicate aliquots of $3 \cdot 10^{7}$ cells containing $10^{-1}$ to $10^{-6}$ fractions of S. cerevisiae WY3711 were prepared in ten-fold dilutions with $S$. pastorianus A15. Aliquots of $S$. cerevisiae WY3711 and $S$. pastorianus A15 were prepared as positive and negative controls, respectively. DNA was extracted from these aliquots using a YeaStar Genomic DNA kit (Zymo Research, Irvine, CA, USA), and PCR was performed on $50 \mathrm{ng}$ template DNA as described above.

\section{Quantitative PCR to detect presence of deletion in STA1 promoter}

The presence of the STA1 gene was tested with quantitative PCR using the previously published primers Sdia-f and Sdia-r (Brandl 2006). These primers amplify a 79-bp fragment from the STA1 open reading frame. In addition, a new primer pair (STA1_UAS_Q_Fw and STA1_UAS_Rv; Table 2) was designed to detect whether the STA1+ yeast strains had the full promoter sequence. Both primers bind within the 1162-bp region that was found to be deleted in the poorly diastatic strains, and amplify a 223-bp fragment. The qPCR reactions were prepared with PerfeCTa SYBR® Green SuperMix (QuantaBio, Beverly, MA, USA) and $0.3 \mu \mathrm{M}$ of the primers (Table 2). The qPCR reactions were performed on a LightCycler® 480 II instrument (Roche Diagnostics, Basel, Switzerland) in two technical replicates on $50 \mathrm{ng}$ template DNA extracted with a YeaStar Genomic DNA kit (Zymo Research, Irvine, CA, USA). The following programme was used: pre-incubation $\left(95{ }^{\circ} \mathrm{C}\right.$ for $\left.3 \mathrm{~min}\right)$, amplification cycle repeated 45 times $\left(95^{\circ} \mathrm{C}\right.$ for $15 \mathrm{~s}, 60^{\circ} \mathrm{C}$ for $30 \mathrm{~s}, 72^{\circ} \mathrm{C}$ for $20 \mathrm{~s}$ with a single fluorescence measurement), melting curve programme $\left(65-97^{\circ} \mathrm{C}\right.$ with continuous fluorescence measurement), and finally a cooling step to $40{ }^{\circ} \mathrm{C}$.

\section{CRISPR/Cas9 mediated deletions}

In order to confirm the effect of the 1162-bp deletion that was observed in the STA1 promoter of the poorly diastatic strains, reverse engineering in the highly diastatic $S$. cerevisiae WY3711 strain was performed using the CRISPR/Cas9 
Table 2 Oligonucleotides used in the study

\begin{tabular}{|c|c|c|}
\hline Name & Sequence $\left(5^{\prime}>3^{\prime}\right)$ & Reference \\
\hline \multicolumn{3}{|c|}{ Primers used for amplification and sequencing of STA1 ORF and upstream region } \\
\hline STA1_Full_Fw & TGGAATGAACAGCGCCAAGT & This study \\
\hline STA1_Full_Rv & AGTGGGAGAAAAAGGTGGCC & This study \\
\hline STA1_24_F & AATGAACAGCGCCAAGTAGC & This study \\
\hline STA1_546_R & TTGAAAGCTATGTGCAGTTGG & This study \\
\hline STA1_482_F & TGTCCCCTAATGTATCCCTCA & This study \\
\hline STA1_1148_R & AAATCTTACCCGTGGATCCTTT & This study \\
\hline STA1_1055_F & CCCAAAATTCATTCGTAGCC & This study \\
\hline STA1_1768_R & TATGCGATGTCCCAGTACGA & This study \\
\hline STA1_1671_F & TGTCAGGCATTGCACAAACT & This study \\
\hline STA1_2384_R & CAATTGAGAACCCTTCAACAA & This study \\
\hline STA1_2267_F & AGGGCAGTTTTATTTACCTTAACA & This study \\
\hline STA1_2951_R & AAGTGGTTGTTGATTCCGATG & This study \\
\hline STA1_2901_F & TCCATGTTCAACCAGTCCAA & This study \\
\hline STA1_3550_R & CTGTCGCTGGAGCCACTC & This study \\
\hline STA1_3474_F & CTTGATGAATGGGACAGTGG & This study \\
\hline STA1_4169_R & GACCGTTCTGAGGCGTTAAA & This study \\
\hline STA1_4051_F & TGGAATTCTTCCGGATTTGA & This study \\
\hline STA1_4746_R & GGTTTGATTGAAGGCAGGTG & This study \\
\hline STA1_4657_F & AGCGAGCTGGTATTCTCCAA & This study \\
\hline STA1_5201_R & ACACGCTTTGGACATCATCA & This study \\
\hline \multicolumn{3}{|c|}{ Primers used for the detection of STA 1} \\
\hline SD-5A & CAACTACGACTTCTGTCATA & $\begin{array}{l}\text { Yamauchi et al. } \\
1998\end{array}$ \\
\hline SD-6B & GATGGTGACGCAATCACGA & $\begin{array}{l}\text { Yamauchi et al. } \\
1998\end{array}$ \\
\hline \multicolumn{3}{|c|}{ Primers used for the detection of intact $S T A 1$ promoter } \\
\hline STA1_UAS_Fw & CCTGGCTCAAATTAAACTTTCG & This study \\
\hline STA1_UAS_Rv & ACCACCAATAGGCAATAGAAA & This study \\
\hline STA1_UAS_Q_FW & CAAGGCAATCAGTTAAAAGA & This study \\
\hline \multicolumn{3}{|c|}{ Primers used in quantitative PCR } \\
\hline Sdia-f & TTCCAACTGCACTAGTTCCTAGAGG & Brandl 2006 \\
\hline Sdia-r & GAGCTGAATGGAGTTGAAGATGG & Brandl 2006 \\
\hline UBC6_F & GATACTTGGAATCCTGGCTGGTCTGTCTC & Teste et al. 2009 \\
\hline UBC6_R & AAAGGGTCTTCTGTTTCATCACCTGTATTTGC & Teste et al. 2009 \\
\hline ALG9_F & CACGGATAGTGGCTTTGGTGAACAATTAC & Teste et al. 2009 \\
\hline ALG9_R & TATGATTATCTGGCAGCAGGAAAGAACTTGGG & Teste et al. 2009 \\
\hline \multicolumn{3}{|l|}{ CRISPR/Cas9 repair oligos } \\
\hline $\begin{array}{l}\text { repair_oligo_promoter_ } \\
\text { deletion }\end{array}$ & $\begin{array}{l}\text { GACGGGGTATTATGAATAAAGGATCCACGGGTAAGATTTGCTGCGCTCTCTTCTAGTTCAAG } \\
\text { AACGGATAACTCATAGAC }\end{array}$ & This study \\
\hline $\begin{array}{l}\text { repair_oligo_orf_ } \\
\text { deletion }\end{array}$ & $\begin{array}{l}\text { GACGGGGTATTATGAATAAAGGATCCACGGGTAAGATTTGACAAAAAAAAATAAAA } \\
\text { GAAAAGCGAGAAGTATACACAAGT }\end{array}$ & This study \\
\hline
\end{tabular}

system. Plasmid construction was carried out using the plasmid pCC-036 as backbone (Rantasalo et al. 2018). pCC-036 contains yeast codon-optimized Cas9 expressed under $T D H 3 p$, guiding RNA (gRNA) expressed under SNR52p, and $h y g R$ for selection on hygromycin. The gRNA protospacer sequence, TGGCTCAAATTAAACTTTCG, was designed using CCTop (Stemmer et al. 2015). The protospacer sequence was designed to induce a doublestranded break within the region to be deleted and was chosen for minimal off-target activity. A synthetic DNA fragment with the gRNA sequence was ordered from Integrated DNA Technologies (Leuven, Belgium) as a gBlock and introduced 
into the plasmid with restriction enzyme-based techniques (Thermo Scientific, Vantaa, Finland). The ligated plasmid was transformed into E. coli TOP10 by electroporation, and plasmid correctness was confirmed by Sanger sequencing. An 80-bp repair oligo (repair_oligo_promoter_deletion), consisting of adjacent 40-bp sequences homologous to those up- and downstream of the deleted region (Table 2), was also ordered from Integrated DNA Technologies (Leuven, Belgium).

Transformation of S. cerevisiae WY3711 was performed using a standard lithium acetate-based protocol with 40-min incubation at $42{ }^{\circ} \mathrm{C}$ (Gietz and Woods 2002). Cells were transformed together with $3.6 \mu \mathrm{g}$ of purified plasmid and $2.5 \mathrm{nmol}$ of repair oligo (double-stranded). The transformed cells were selected on plates containing $300 \mathrm{mg} / \mathrm{L}$ Hygromycin B (Sigma-Aldrich, Espoo, Finland). Colony PCR using primer pairs 1055F/2951R and STA1_UAS_Fw/STA1_UAS_Rv, and Sanger sequencing, was used to confirm successful deletion in the transformed cells. Colonies from selection plates were replated three times onto YPD agar plates to encourage plasmid loss, after which they were stored at $-80^{\circ} \mathrm{C}$.

The same CRISPR/Cas9 plasmid that was used above to delete the 1162-bp region in the STA1 promoter, was also used to delete the entire STA1 open reading frame from three of the highly diastatic S. cerevisiae strains (TUM PI BA 109, TUM 71 , and WY3711). An 80-bp repair oligo (repair_oligo_orf_deletion; Table 2), consisting of adjacent 40-bp sequences homologous to those up- and downstream of the region to be deleted $(-1370$ to +2421$)$ was transformed together with the purified plasmid as described above. Colony PCR using primer pairs STA1_Full_Fw/STA1_Full_Rv, 1055F/5201R, and SD-5A/SD-6B, and Sanger sequencing, was used to confirm successful deletion in the transformed cells (Supplementary Fig. S1).

\section{STA1 transcript analysis by RT-qPCR}

Strains were grown overnight in YP-Glucose, after which four replicate cultures per strain were started by inoculating $20 \mathrm{~mL}$ YPGE (1\% yeast extract, $2 \%$ peptone, $3 \%$ glycerol, and $2 \%$ ethanol) to a starting OD600 of 0.1 . Cultures were overnight at $25^{\circ} \mathrm{C}$ (OD600 varied between 2 and 4), after which RNA was isolated from pelleted yeast using hot formamide extraction (Shedlovskiy et al. 2017). RNA was precipitated by first diluting $50 \mu \mathrm{L}$ RNA solution with $25 \mu \mathrm{L} 3 \mathrm{M}$ sodium acetate and $200 \mu \mathrm{L}$ nuclease-free water, after which $825 \mu \mathrm{L}$ ice-cold ethanol was added and solutions were stored overnight at -20 ${ }^{\circ} \mathrm{C}$. The solutions were centrifuged at $16000 \times \mathrm{g}$ for $30 \mathrm{~min}$, after which the pellet was washed with ice-cold $75 \%$ ethanol and tubes were centrifuged again at $8000 \times g$ for $5 \mathrm{~min}$. The supernatant was removed and the pellet was allowed to air-dry for up to $30 \mathrm{~min}$, after which the RNA pellet was dissolved in nuclease-free water. The RNA solution was treated with
TURBO DNAse (DNA-Free kit, Invitrogen, Carlsbad, CA, USA) and the DNAse was subsequently inactivated using the supplied inactivation reagent. RNA was quantified with a Qubit 2.0 fluorometer and its quality was assessed on $1.2 \%$ agarose gels (made in $1 \times$ TAE buffer).

$250 \mathrm{ng}$ of total RNA was reverse-transcribed using a qScript Flex cDNA kit (QuantaBio, Beverly, MA, USA), using a mixture of supplied oligo-dT and random primers according to kit instructions. The resulting cDNA was diluted 5 -fold and $4 \mu \mathrm{L}$ of diluted cDNA (corresponding to $10 \mathrm{ng}$ of total RNA) was used as template in $20 \mu \mathrm{L}$ qPCR reactions. The qPCR reactions were prepared with PerfeCTa SYBR ${ }^{\circledR}$ Green SuperMix (QuantaBio, Beverly, MA, USA) and $0.3 \mu \mathrm{M}$ of gene-specific primers (Table 2). In addition to using primers specific to $S T A 1$, reactions with primers for two house-keeping genes ( $A L G 9$ and $U B C 6$ ) were also performed (Teste et al. 2009). The qPCR reactions were performed on a LightCycler® 480 II instrument (Roche Diagnostics, Basel, Switzerland) in two technical replicates on the reversetranscribed RNA isolated from four biological replicates. The following programme was used: pre-incubation $\left(95^{\circ} \mathrm{C}\right.$ for $3 \mathrm{~min}$ ), amplification cycle repeated 45 times $\left(95^{\circ} \mathrm{C}\right.$ for 15 $\mathrm{s}, 60^{\circ} \mathrm{C}$ for $30 \mathrm{~s}, 72{ }^{\circ} \mathrm{C}$ for $20 \mathrm{~s}$ with a single fluorescence measurement), melting curve programme $\left(65-97{ }^{\circ} \mathrm{C}\right.$ with continuous fluorescence measurement), and finally a cooling step to $40{ }^{\circ} \mathrm{C}$. The relative expression of STA1 in the three examined yeast strains was calculated using the 'delta-delta $\mathrm{C}_{\mathrm{T}}$ '-method by normalizing expression to that of the two house-keeping genes $A L G 9$ and UBC6 (Pfaffl 2001).

\section{Oxford Nanopore MinION whole genome sequencing}

The genomes of $S$. cerevisiae WY3711 and A62 were sequenced using an Oxford Nanopore MinION. Genomic DNA was extracted with a YeaStar Genomic DNA kit (Zymo Research, Irvine, CA, USA) and then barcoded with the Native Barcoding kit (EXP-NBD104; Oxford Nanopore Technology, United Kingdom). A 1D sequencing library was then prepared from the barcoded DNA using the Ligation Sequencing Kit (SQK-LSK109; Oxford Nanopore Technology, United Kingdom). The library was sequenced on a FLO-MIN106D (R9.4.1) flow cell with a MinION device (Oxford Nanopore Technology, United Kingdom). The raw fast5 files were basecalled using the GPU-version of Guppy (version 2.3.7; using the supplied dna_r9.4.1_450bps_flipflop.cfg configuration). The basecalled reads were demultiplexed with qcat (version 1.0.1; available from https://github.com/nanoporetech/qcat), and filtered to a minimum length of $1000 \mathrm{bp}$ and average read quality score of 10 using NanoFilt (version 2.2.0; available from https://github.com/wdecoster/nanofilt). This resulted in a total of $1.3 \mathrm{Gbp}$ of sequence for S. cerevisiae 
WY3711 (coverage of $108 \times$ ) and 1.9 Gbp of sequence for $S$. cerevisiae A81062 (coverage of $155 \times$ ).

\section{De novo assembly}

The sequencing reads generated in this study (for $S$. cerevisiae WY3711 and S. cerevisiae A62) were de novo assembled using the LRSDAY (version 1.4) pipeline (Yue and Liti 2018). The initial assemblies were produced with smartdenovo (available from https://github.com/ruanjue/ smartdenovo) using default settings. The assemblies were then polished twice with Nanopolish $(0.11 .1$; available from https:/github.com/jts/nanopolish). Alignment of long reads was performed with minimap2 (version 2.16; Li 2018). The contigs in the polished assemblies were then scaffolded with Ragout (version 2.0; Kolmogorov et al. 2014) to $S$. cerevisiae S288C (R64-2-1). Assembly statistics are available in Supplementary Table S1 and Supplementary Fig. S2, while assemblies are available as Supplementary Data 2-3.

In addition, de novo assembly of long sequencing reads produced in other studies (accession numbers are available in Supplementary Table S2) was also carried out using the LRSDAY pipeline essentially as described above. The assemblies were polished twice with Pilon (version 1.22; Walker et al. 2014) using Illumina reads (accession numbers are available in Supplementary Table S2). Identity between assemblies was determined with 'dnadiff' from MUMmer (version 3.23; Kurtz et al. 2004).

\section{Prevalence of STA1 and phylogenetic analysis}

In addition to the two genome assemblies produced from sequencing reads generated in this study, we retrieved publically available genome assemblies of $S$. cerevisiae. Genome assemblies of the $157 \mathrm{~S}$. cerevisiae strains described in Gallone et al. (2016) were retrieved from NCBI (BioProject PRJNA323691). Genome assemblies of the 1011 $S$. cerevisiae strains described by Peter et al. (2018) were retrieved from https://www.yeastgenome.org/1011-yeastgenomes. The genome assembly of $S$. cerevisiae A81062 (Krogerus et al. 2016) was retrieved from NCBI (Assembly ASM193724v1). The prevalence of the STA1 gene in natural $S$. cerevisiae isolates was investigated by performing a BLAST search of sequence 'STA1_BLAST' (Supplementary Table S3) in the collected genome assemblies using NCBI-BLAST (version 2.6.0).

Multiple sequence alignment of the $S$. cerevisiae assemblies was performed with the NASP pipeline (Sahl et al. 2016) using $S$. cerevisiae S288C (R64-2-1) as the reference genome. A matrix of single nucleotide polymorphisms (SNP) in the 1171 strains was extracted from the aligned sequences. The SNPs were annotated with SnpEff (Cingolani et al. 2012) and filtered as follows: only sites that were in the coding sequence of genes, present in all 1171 strains and with a minor allele frequency greater than $0.25 \%$ were retained (in at least 3 strains). The filtered matrix contained 26725238 SNPs (462,842 sites). A maximum-likelihood phylogenetic tree was estimated using IQ-TREE (Nguyen et al. 2015). IQTREE was run using the 'GTR+F+R4' model and 1000 ultrafast bootstrap replicates (Minh et al. 2013). The resulting maximum-likelihood tree was visualized in FigTree and rooted with the Taiwanese outgroup.

\section{Copy number variation of STA1}

The copy number and zygosity of the STA 1 gene in strains of the 'Beer 2'/'Mosaic Beer' lineage were estimated from read coverage using publically available short-read sequence data. Sequence reads were obtained from NCBI-SRA (accession numbers listed in Supplementary Table S4), and trimmed and filtered with Trimmomatic (version 0.36) (Bolger et al. 2014). As STA1 is missing from the $S$. cerevisiae S288C (R642-1) reference genome, the sequence of the STA 1 open reading frame and promoter (Supplementary Data 1) were concatenated to the S288C reference genome as a separate contig. Reads were then aligned to this concatenated reference genome using BWA-MEM (version 0.7.17). The alignments were filtered to a minimum MAPQ value of 50 (to remove reads mapping to both STA1 and either FLO11 or SGA1). Because of the sequence identity between STA1 and both FLOII and SGA1, the copy number and zygosity of STA 1 could not be estimated from the coverage across the whole gene. Instead, two unique regions in the $S T A 1$ open reading frame $(+65$ to +143 relative to $S T A 1$ start codon) and within the deleted region of the STA 1 promoter ( -923 to -823 relative to STA1 start codon) were identified. Copy numbers and zygosity were then estimated by comparing the median read coverage of these two unique regions in STA1 with that of the whole genome. The read coverage of the alignments was calculated with mosdepth (vesrion 0.2.6; Pedersen and Quinlan 2018).

\section{Data visualization and analysis}

Data and statistical analyses were performed with R (http:// www.r-project.org/). Plots were produced in $\mathrm{R}$ and FigTree.

\section{Data availability}

The Sanger sequences generated in this study are available in the Supplementary material, and the long sequencing reads have been submitted to NCBI-SRA under BioProject number PRJNA544899 in the NCBI BioProject database (https:// www.ncbi.nlm.nih.gov/bioproject/). 


\section{Results}

\section{Screening of diastatic ability}

The diastatic ability of eighteen $S$. cerevisiae and one S. pastorianus strain (Table 1) was tested using three different tests: growth in beer, growth on starch agar, and fermentation of dextrin as a sole carbon source. Of the eighteen $S$. cerevisiae strains, 15 tested positive for STA1 using the SD-5A/SD-6B primer pair (Yamauchi et al. 1998). Despite carrying the STA1 gene, only five out of 15 strains, were able to grow in beer and on starch agar (Fig. 1a). These five strains also fermented dextrin efficiently. All four of the strains that tested negative for STAl were unable to grow in beer or on starch agar and did not consume any dextrin.

We then amplified and sequenced the STA1 open reading frame and a $2.5 \mathrm{~kb}$ upstream region, to search for polymorphisms that might explain the variation in diastatic ability in the 15 strains that tested positive for STA1. No nonsense mutations in the open reading frame of STA1 were observed in any of the strains. However, we did observe a 1162-bp deletion upstream of STA1 (-1370 to -209 from the start codon) in 10 out of the 15 strains (Fig. 2 and Fig. 1a). This 1162-bp region contains an upstream activation sequence and transcription factor (Ste12 and Tec1) binding site (Kim et al. 2004a, b). Interestingly, the presence of this deletion appeared to coincide with decreased diastatic ability (Fig. 1). The strains with the deletion in the promoter exhibited significantly less diastatic ability in all tests (Fig. 1b-e). We hypothesized that this deletion impedes transcription of STA1, which in turn decreases the amount of STA1-derived glucoamylase produced by the strains.

\section{Confirmation by reverse engineering}

To confirm that the 1162-bp deletion that was observed in the STA1 promoter had a negative effect on diastatic ability and STA1 expression, we performed reverse engineering aided by the CRISPR/Cas9 system. The gRNA protospacer sequence was designed to cause a double-stranded break within the region to be deleted (Fig. 2e). The 1162-bp region (- 1370 to - 209 upstream of the STA1 open reading frame) was deleted from the highly diastatic WY3711 strain by transformation with a Cas9- and gRNA-expressing plasmid and an 80-bp double-stranded repair oligo. PCR using primer pairs binding within (STA1_UAS_Fw / STA1_UAS_Rv) and outside the deleted region (1055F/2951R), together with Sanger sequencing, was used to confirm successful deletion (Fig. $2 \mathrm{~d}$ and Supplementary Fig. S1).

The deletion strain, WY3711 D1, was then tested for diastatic ability. WY3711, the wild-type strain from which WY3711 D1 was derived, and WLP570, a strain that naturally contains the $1162 \mathrm{bp}$ deletion in the promoter, were included as controls. In all tests, the diastatic ability of WY3711 D1 was less than WY3711. When inoculated into beer, WY3711_D1 and WLP570 grew significantly less than WY3711 (Fig. 3a). In contrast to WY3711, WY3711 D1 and WLP570 had also not consumed any dextrin from the beer after 3 weeks (Fig. 3d). When the strains were grown in minimal media with dextrin as the sole carbon source, we saw negligible dextrin consumption after 3 weeks with WY3711_D1 and WLP570 when cultures were incubated anaerobically (Fig. 3b). Interestingly, we observed delayed activity in these strains when cultures were incubated aerobically (Fig. 3c). WY3711 performed similarly in both anaerobic and aerobic conditions, and in both cases consumed significantly more dextrin than the strains with the deletion in the promoter.

To test the effect that the 1162 bp deletion in the STA1 promoter has on the expression of STA1, we performed RTqPCR analysis on RNA extracted from exponential phase cultures in YPGE. This growth medium was chosen as glucose represses STA1 expression (Pretorius et al. 1986; Kim et al. 2004b). We observed a 150-200 fold higher abundance of STA1 mRNA in the samples from WY3711 compared to WY3711_D1 and WLP570, respectively (Fig. 3e). These results suggest that the decreased diastatic ability observed in the STA1+ strains with the 1162-bp deletion is a result of decreased expression of STA 1 .

\section{Designing primers for the detection of the full STA1 promoter}

To improve the reliability of the molecular detection methods for diastatic $S$. cerevisiae, we designed two new primer pairs that bind within the 1162 bp region that is absent in the poorly diastatic STA1+ strains. These new primers can therefore be used to differentiate whether a strain that has tested positive for STA1 with the SD-5A / SD-6B primers (Yamauchi et al. 1998) is likely to be highly diastatic or not. The first primer pair (STA1_UAS_Fw / STA1_UAS_Rv) was designed to produce a $599 \mathrm{bp}$ amplicon, and can be used together with the SD-5A / SD-6B primers in a single multiplex PCR reaction (Fig. 2b, c). Here, strains with the full STA1 promoter produce two amplicons (599 and 868 bp), while poorly diastatic strains only produce a single amplicon ( $868 \mathrm{bp}$ ). To test how this multiplex PCR reaction would perform in a simulated brewery scenario, we contaminated a lager yeast culture with varying ratios of diastatic S. cerevisiae WY3711 $\left(10^{-6}-10^{-1}\right)$. We were able to detect the presence of diastatic $S$. cerevisiae at a concentration of $10^{-4}$ (Fig. 4a).

In addition, a second primer pair (STA1_UAS Q Fw / STA1 UAS Rv) was also designed to be useable in quantitative PCR reactions where a shorter amplicon is desired. This primer pair produces a 223-bp amplicon. We performed quantitative PCR reactions on DNA extracted from $S$. cerevisiae 
a

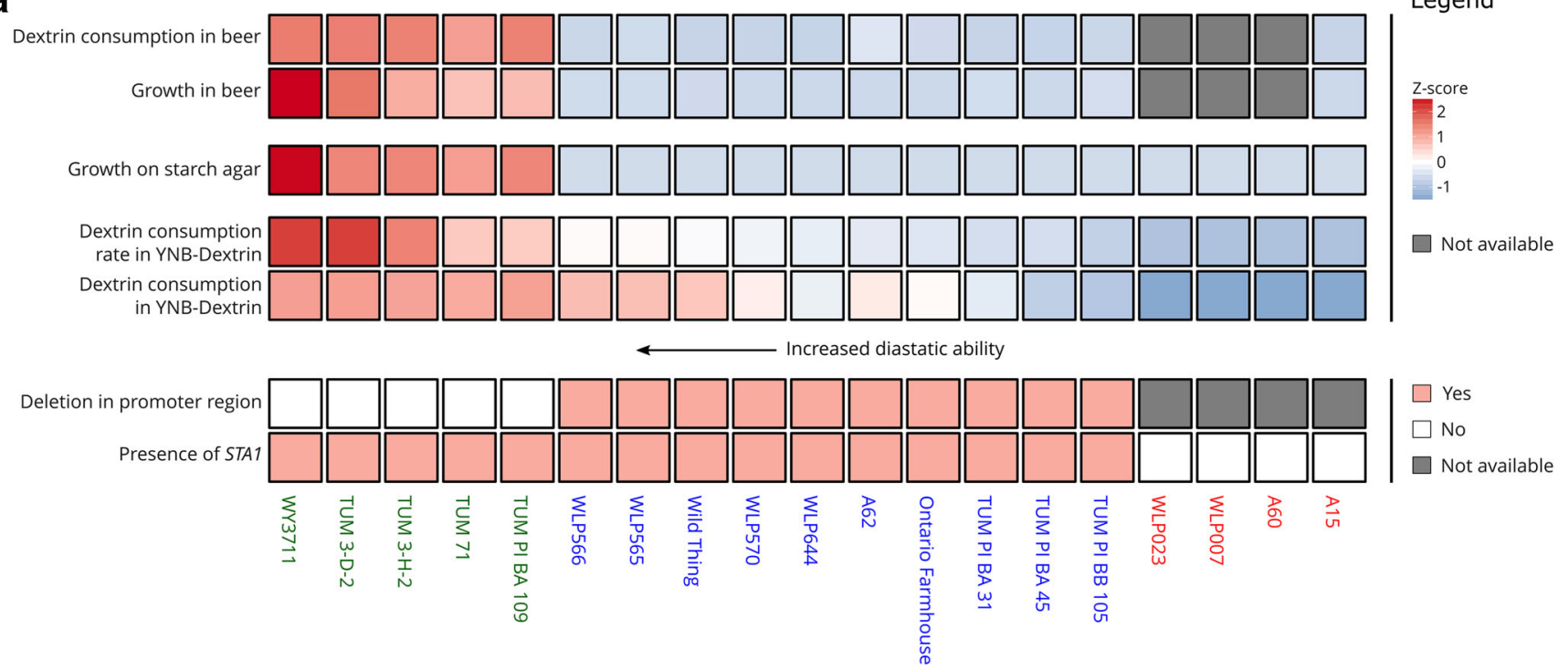

b
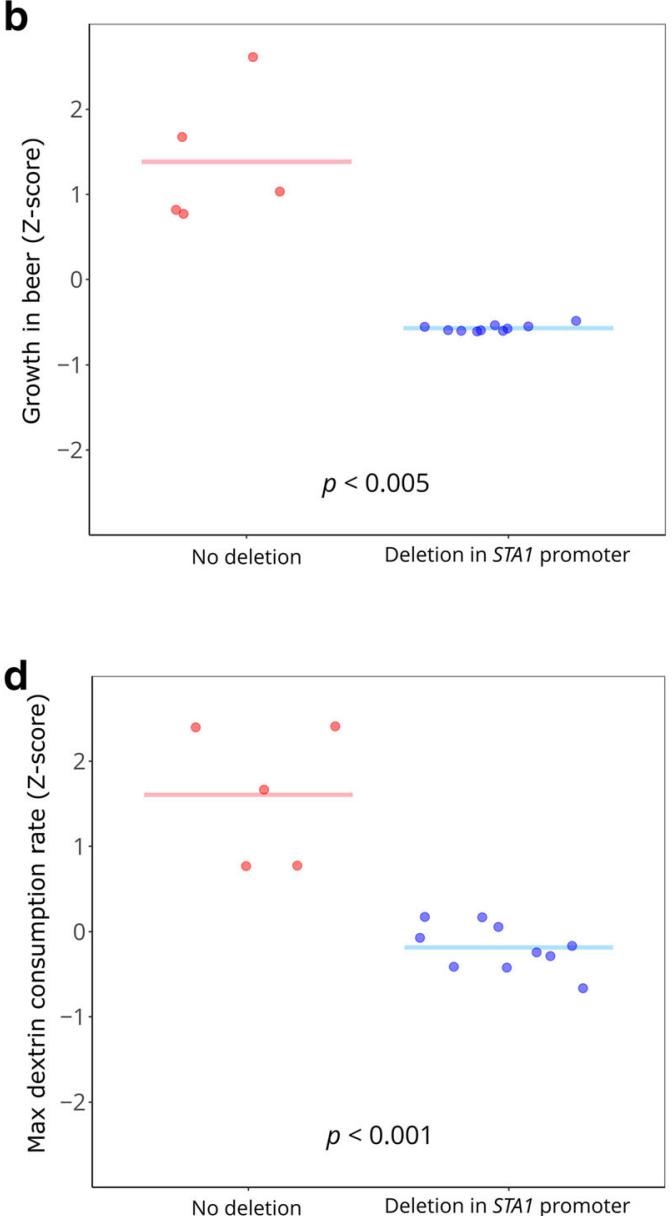

Fig. 1 A comparison of the diastatic ability of 15 STA1+ and 4 STA1yeast strains. a A heatmap of the diastatic ability based on three different tests in the 19 Saccharomyces strains. The heatmap is coloured based on $Z$-scores (red and blue indicate values higher and lower than average, respectively). The strains are coloured as follows; green: $S T A 1+$, no deletion in STA1 promoter; blue: STA1+, deletion in STA1 promoter;
C

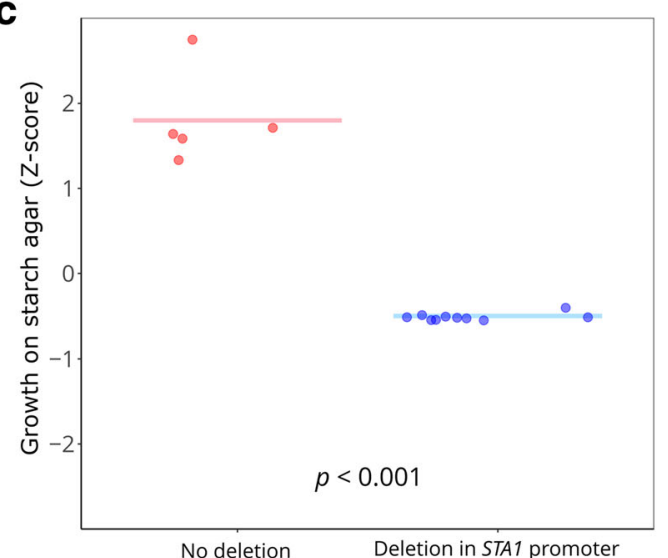

e

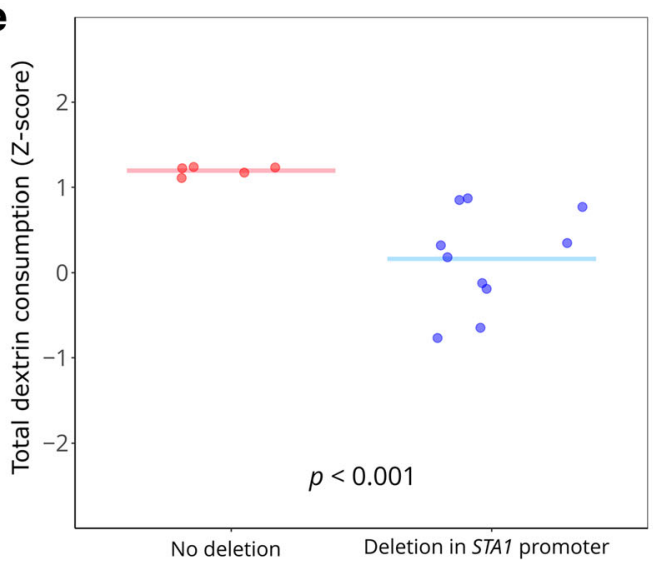

red: STA1-. b-e Pairwise comparison of the results from the individual diastatic tests (b: growth in beer; c: growth on starch agar; d-e: fermentation of dextrin) between $S T A 1+$ strains with no deletion in the STA1 promoter compared to strains with a deletion in the STA1 promoter. The statistical significance between the two groups was tested with the Mann-Whitney $U$ test 

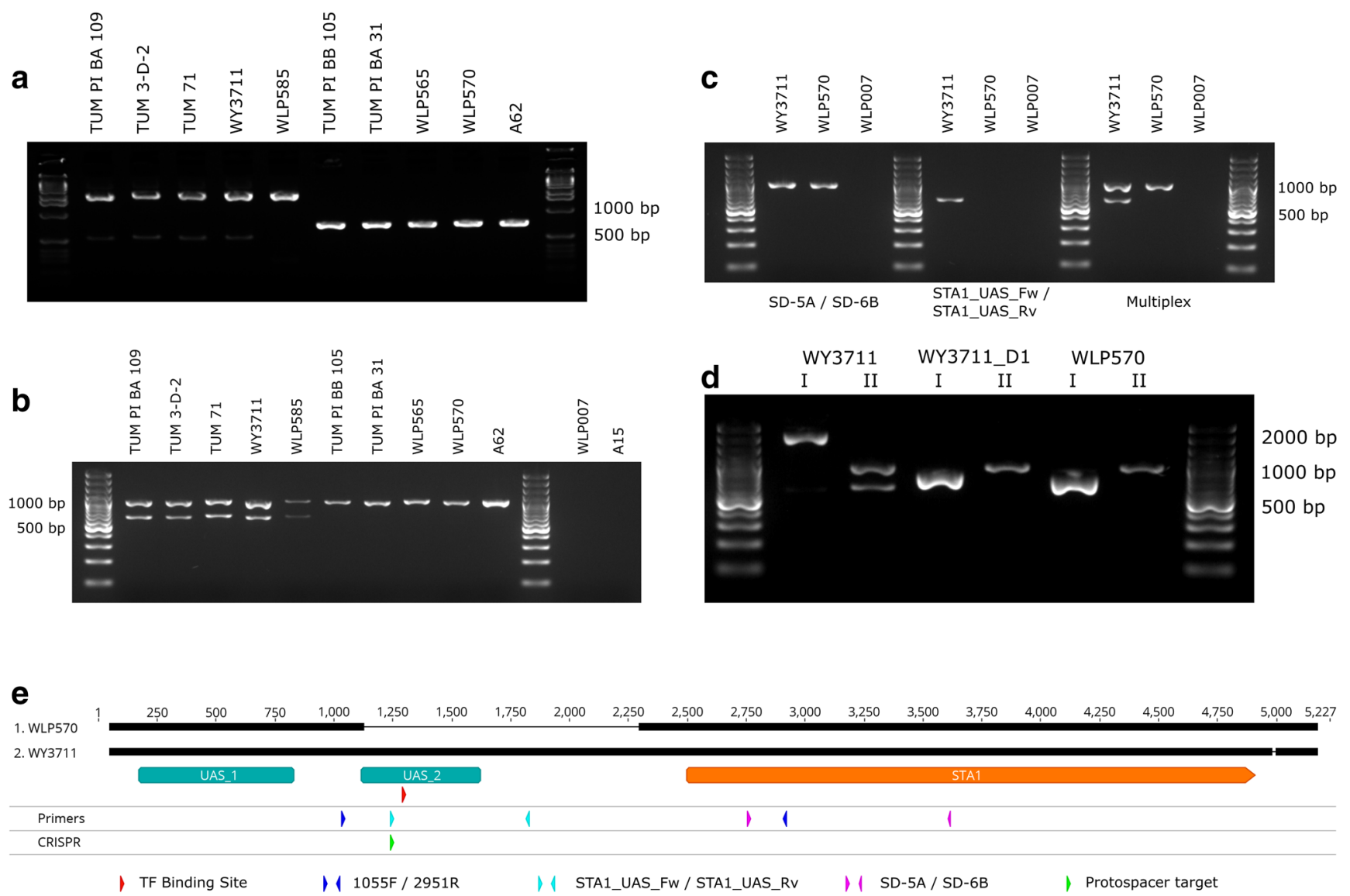

Fig. 2 The 1162-bp deletion in the STA1 promoter. a PCR products from 10 STA1+ strains using primers STA1_1055_F/ STA1_2951_R. b Multiplex PCR products from the same 10 STA $1+$ strains as (a) and two STA1- controls using primers SD-5A/SD-6B and STA1_UAS_Fw/ STA1_UAS_Rv. c Individual and multiplex PCR reactions with WY3711 (STA1+, no deletion in STA1 promoter), WLP570 (STA1+, deletion in STA1 promoter), and WLP007 (STA1-) using primers SD-5A/SD-6B and STA1_UAS_Fw/STA1_UAS_Rv. d Confirmation of successful deletion

WY3711, S. cerevisiae WLP570 and S. pastorianus A15 using both the previously published Sdia-f/Sdia-r primer pair that bind within the STA1 ORF (Brandl 2006), and our newly designed primer pair that bind within the STA1 promoter. As expected, amplification using the Sdia-f / Sdia-r primer pair was observed for both WY3711 and WLP570 (threshold cycles around 17-19), while no signal above the background was observed for the negative control A15 after 40 cycles (Fig. 4b). With the new primer pair on the other hand, amplification was only observed for the highly diastatic WY3711, while no signal above the background was observed for the poorly diastatic WLP570 and the negative control A15 after 40 cycles. We also tested the DNA extracted from the lager yeast cultures contaminated with varying ratios of $S$. cerevisiae WY3711 $\left(10^{-6}-10^{-1}\right)$ using the same quantitative PCR reactions. Both primer pairs produced similar linear responses in regard to increasing threshold cycle $\left(C_{\mathrm{t}}\right)$ values as the ratio of WY3711 in the yeast culture decreased 10-fold (Fig. 4c). No signal above the background was observed from the yeast culture contaminated with $10^{-6}$ of of the 1162-bp region in the STA1 promoter in strain WY3711 D1 using primers STA1_1055_F/STA1_2951_R (represented by I) and the multiplex primers SD-5A/SD-6B and STA1_UAS_Fw/STA1_UAS_Rv (represented by II). e Sequence alignment of $S T \bar{A} 1$ open reading frame and upstream region from WY3711 and WLP570, and locations of PCR primers, transcription factor binding site (TF) and CRISPR protospacer target. UAS_1 and UAS_2 are locations of upstream activation sequences described in Kim et al. (2004a, b)

S. cerevisiae WY3711 after 40 cycles with either of the primer pairs, but the presence of diastatic S. cerevisiae could be detected at a concentration of $10^{-5}$.

\section{Prevalence of STA1}

To determine how common the STA 1 gene is among wild and domesticated strains of $S$. cerevisiae, a BLAST search was performed in the genome assemblies produced in recent whole genome sequencing studies (Gallone et al. 2016; Peter et al. 2018). Because of the chimeric nature of STA1 (rearranged fragments from $F L O 11$ and $S G A 1$ ), genomes assembled from short reads have difficulty capturing the full STA 1 sequence on a single contig. To demonstrate, the short-read genome assemblies of three STA1+ strains (WLP570, OS899, and A81062) were obtained (Krogerus et al. 2016; Peter et al. 2018), and these were searched for the STAl sequence (GenBank X02649.1) using BLAST. None of the three assemblies contained the full STA1 sequence on a single contig 

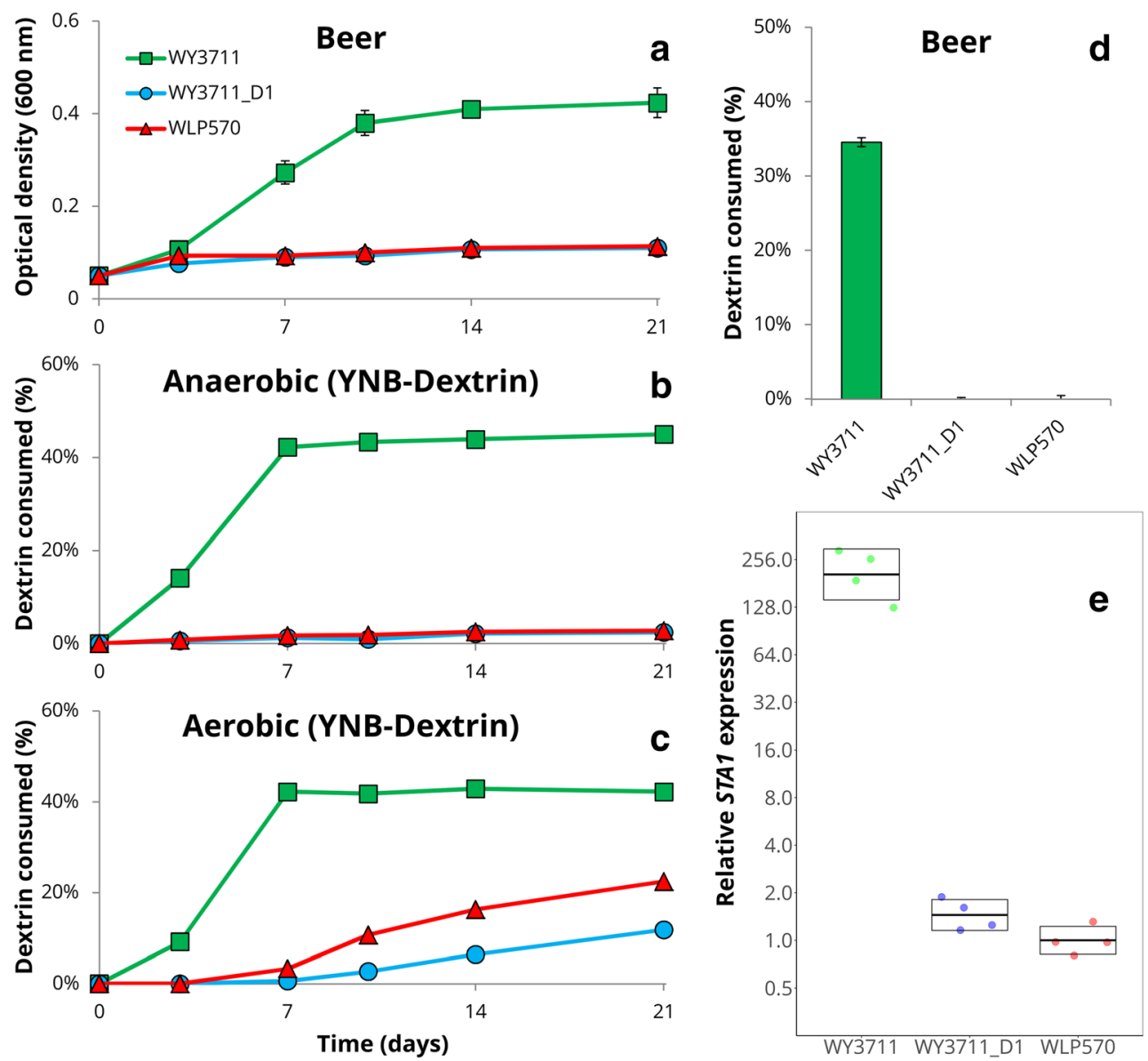

Fig. 3 Confirmation of the role of the 1162-bp deletion in the STA1 promoter by reverse engineering. Three STA1+ strains were compared: WY3711 (no deletion in STA1 promoter), WY3711 D1 (CRISPR-mediated deletion in STA1 promoter), and WLP570 (natural deletion in STA1 promoter). a The optical density $(600 \mathrm{~nm})$ when strains were inoculated into beer at a starting value of 0.1 . Values for WY3711 were different from the two other strains starting from day 7 ( $p<0.01$ by two-tailed Student's $t$ test). b, $\mathbf{c}$ The amount of dextrin consumed from YNB-Dextrin media in anaerobic (b) and

aerobic (c) conditions. Values for WY3711 were different from the two other strains starting from day 4 ( $p<0.01$ by two-tailed Student's $t$-test). d The amount of dextrin consumed from the beer (a) after 3 weeks of incubation. $\mathbf{e}$ The relative expression of STA1 (normalized to $A L G 9$ and UBC6) determined by RT-qPCR in derepressed conditions. Points indicate values from four biological replicates and boxes indicate the mean and standard deviation. Values for WY3711 were significantly higher than those of the two other strains ( $p<0.01$ by two-tailed Student's $t$ test)

(Supplementary Table S5). In addition to short sequence reads, long sequence reads (Nanopore reads for WLP570 and OS899, and PacBio reads for A81062) are available for all three strains (Krogerus et al. 2016; Istace et al. 2017). These were obtained and de novo assembled using the LRSDAY pipeline (Yue and Liti 2018). The long-read genome assemblies were again queried for the STA1 sequence using BLAST, and now, the full sequence was captured on single contigs in all three strains (Supplementary Table S5).

In light of this, STA1 detection in the short-read genome assemblies was instead carried out by searching for the presence of a 79 bp STA1-specific sequence ('STA1 BLAST' in Supplementary Table S3). This sequence is amplified by the STA1-specific Sdia-f and Sdia-r PCR primers described for the detection of diastatic S. cerevisiae (Brandl 2006). Out of the 1169 publically available genome assemblies that were queried, 54 contained a $100 \%$ match to the full 79-bp sequence (Supplementary Table S6). Interestingly, of these 54 strains,

51 were concentrated in the 'Beer 2' ('Mosaic Beer') and the 'French Guiana human' populations, while the remaining three were described as mosaic (Fig. 5). While the majority of the strains in these two populations were STA1+, not all of them were $(69 \%$ and $63 \%$ in the 'Beer 2' and 'French Guiana human' populations, respectively). The majority of the 'Beer 2' strains were homozygous for the STA1 allele, but a cluster of strains (Beer004, Beer011, Beer039, Beer040, and CFF) appeared to be hemizygous (Supplementary Table S4). An increased copy number of STA 1 was also estimated for multiple strains (AEA, Beer013, Beer059, Beer084, and BRM).

The 54 STA $1+$ genomes were also queried for the presence of the 1162-bp deletion in the STA1 promoter using BLAST ('STA1 deletion BLAST' in Supplementary Table S3). While we observed that the majority of the 15 STA1+ strains screened in this study had a deletion in the STA1 promoter, only ten out of the 54 sequenced STA1+ strains appeared to 
Fig. 4 The sensitivity of the newly designed PCR primers for differentiation of STA1+ strains with and without the 1162-bp deletion in the STA1 promoter. a Multiplex PCR products using primers SD-5A/SD-6B and STA1_UAS_Fw/STA1_UAS_Rv from DNA extracted from duplicate $S$. pastorianus A15 cultures contaminated with increasing tenfold ratios of contamination by S. cerevisiae WY3711 (values $10^{-1}-10^{-6}$ indicate fraction of WY3711 in the culture). b, $\mathbf{c}$ The cycle threshold $\left(C_{\mathrm{t}}\right)$ values of quantitative $\mathrm{PCR}$ reactions using DNA extracted as in (a) using primers STA1_UAS_Q_Fw/ STA1_UAS_Rv and Sdia-f/Sdia-

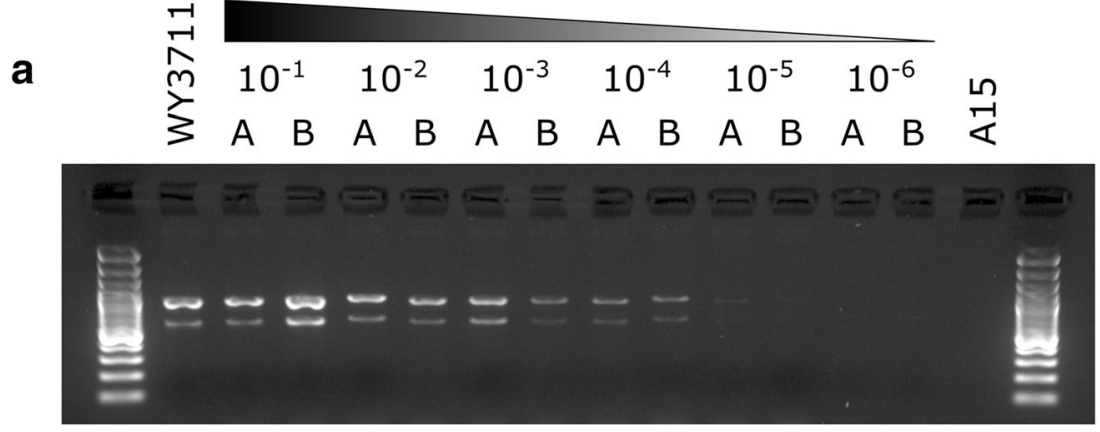

\begin{tabular}{|c|c|c|c|c|}
\hline Strain & STA1 & $\begin{array}{l}\text { Deletion in } \\
\text { promoter }\end{array}$ & $\begin{array}{c}\mathrm{C}_{\mathrm{t}} \text { Value } \\
\text { Sdia-f / Sdia-r }\end{array}$ & $\begin{array}{c}\text { C } \text { Calue }_{\mathrm{t}} \\
\text { STA1_UAS_Q_Fw / } \\
\text { STA1_UAS_Rv }\end{array}$ \\
\hline WY3711 & Yes & No & $17.0( \pm 0.08)$ & $18.4( \pm 0.22)$ \\
\hline WLP570 & Yes & Yes & $18.9( \pm 0.01)$ & $>40$ \\
\hline A15 & No & - & $>40$ & $>40$ \\
\hline
\end{tabular}

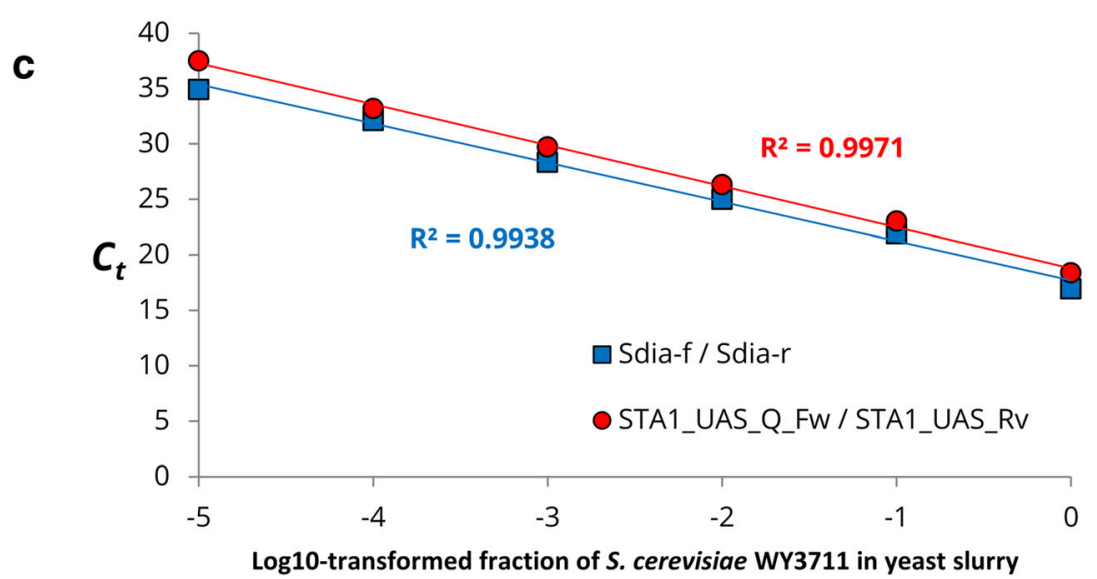

have the $1162 \mathrm{bp}$ deletion in the promoter (Fig. 5). All ten strains belonged to the 'Beer 2' ('Mosaic Beer') population. The deletion in the STA1 promoter was heterozygous in three of the ten strains (Beer013, Beer059, and Beer091), while homozygous in the remaining seven (Supplementary Table S4).

Whole genome sequencing of $S$. cerevisiae WY3711 also confirmed that the strain belongs to the 'Beer 2' ('Mosaic Beer') population (Fig. 5). The sequencing reads generated with the Nanopore MinION are known to be error-prone (Istace et al. 2017), which naturally affects the reliability of the analysis. Therefore we also sequenced $S$. cerevisiae A62 in the same sequencing run to include as a control. The assembly of A62 using only reads generated from the MinION (polishing with NanoPolish, but no polishing with Illumina reads) showed $99.6 \%$ identity with the assembly produced from PacBio reads (polished with Illumina reads), and the assemblies grouped next to each other in the phylogenetic tree (Fig. 5).

\section{STA1 improves maltotriose consumption during wort} fermentations

Strains in the 'Beer 2' population have been shown to utilize maltotriose efficiently, despite carrying frameshift mutations in AGT1/MAL11 (Gallone et al. 2016). It has been suggested that the 'Beer 2' strains instead utilize alternative mechanisms for maltotriose use. The glucoamylase produced from the STA1 gene does not only hydrolyse malto-oligomers efficiently, by cleaving $\alpha-1,4$ bonds, but is also active on maltotriose (Kleinman et al. 1988). We therefore explored the role of STA1 in enabling maltotriose use during wort fermentations.

Initially, we performed fermentations by inoculating 15 ${ }^{\circ}$ Plato all-malt wort with $1 \mathrm{~g} \mathrm{~L}^{-1}$ of WY3711 and WY3711 D1. During the first $24 \mathrm{~h}$ of fermentation, an identical amount of ethanol was produced by both strains (Fig. 6). However, after that time-point, the fermentation with WY3711_D1 slowed considerably. Analysis of the wort sugars during fermentation revealed that maltotriose 


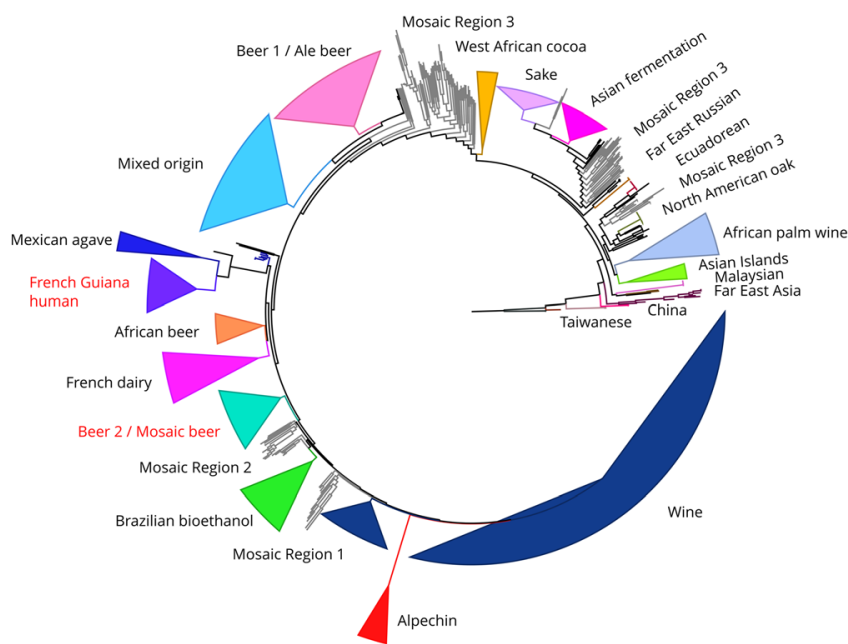

Beer 2 / Mosaic Beer

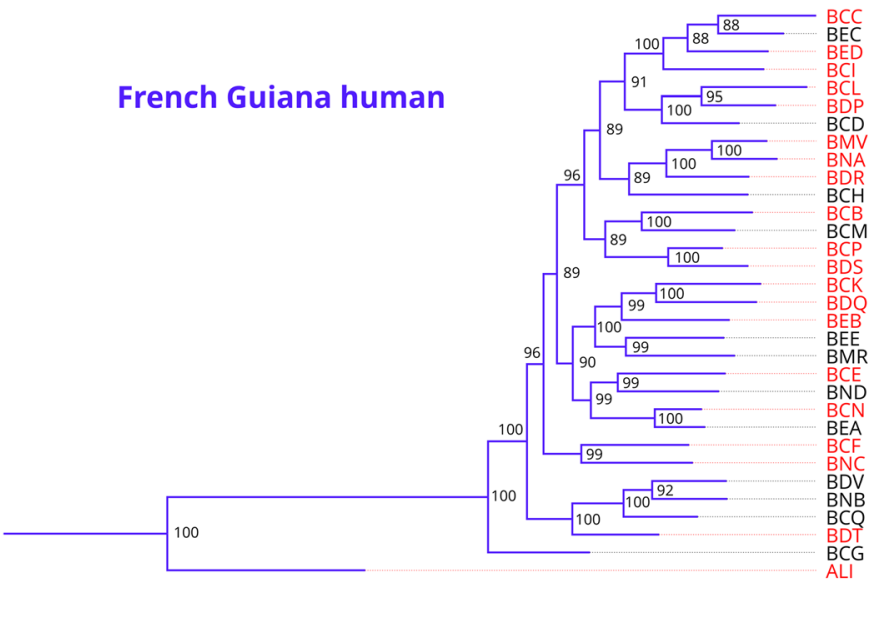

\section{Legend}

STA1 detected in assembly STA1 not detected in assembly $*$ : deletion in STA1 promoter

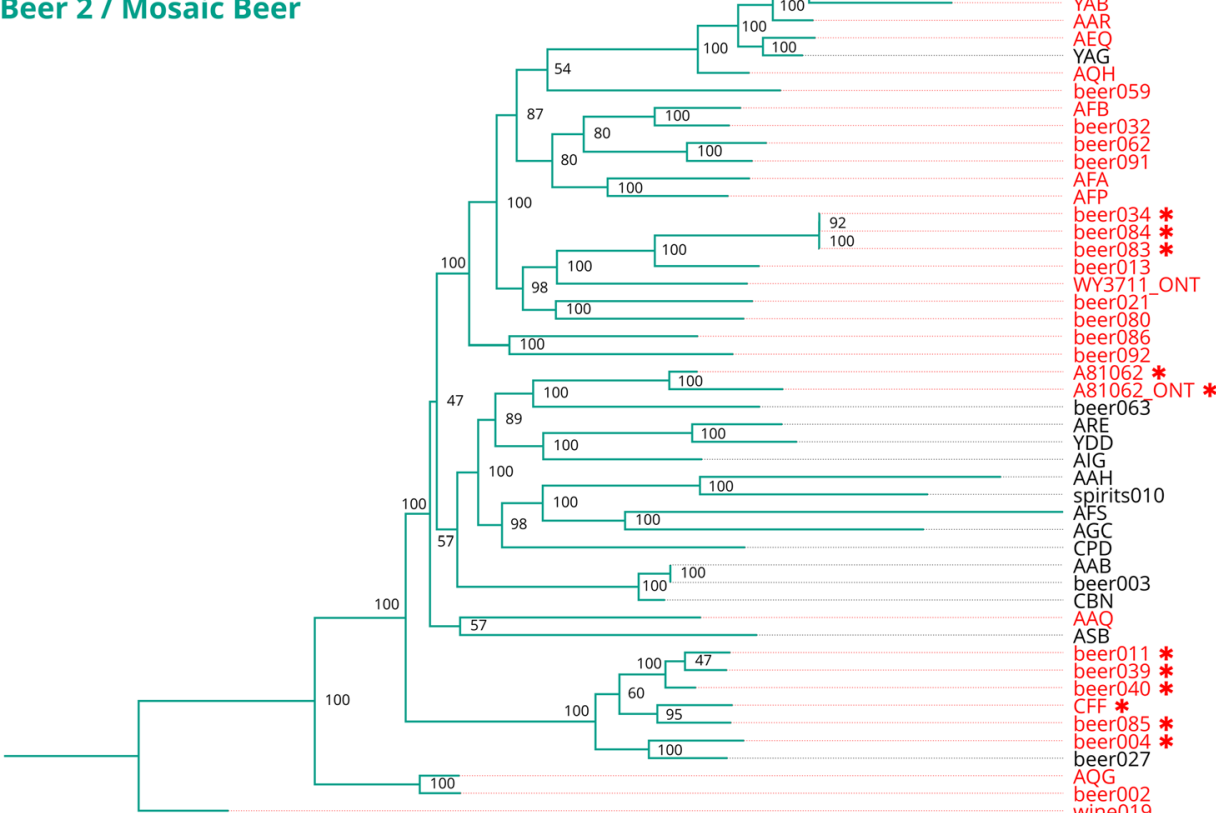

Fig. 5 The prevalence of STA1 in 1171 S. cerevisiae genome assemblies. A maximum-likelihood phylogenetic tree based on 26725238 SNPs at 462842 sites in S. cerevisiae genome assemblies obtained from Gallone et al. (2016), Peter et al. (2018) and Krogerus et al. (2016) (rooted with Taiwanese strains as outgroup). Clades have been collapsed to improve clarity and the names of clades containing STAI+ strains are coloured red ('French Guiana human', and 'Beer 2\%'Mosaic beer'). The 'French

use in particular, was markedly decreased in WY3711_D1 compared to WY3711 (Fig. 6). At $96 \mathrm{~h}$, for example, WY3711 had consumed nearly $80 \%$ of the wort maltotriose, while WY3711 D1 had only consumed $12 \%$. These results suggest that STA1 has a central role in enabling maltotriose use in $S T A 1+$ yeast strains. Interestingly, in contrast to typical wort fermentations with brewing strains, we also detected glucose in the wort throughout most of the active fermentation with the wild-type strain. This was presumably a result of the glucoamylase activity,
Guiana human' and 'Beer 2'/'Mosaic beer' clades have been expanded, and strain names have been coloured red if STA1 was detected in the assembly. A red asterisk depicts a strain with a 1162-bp deletion in the STA1 promoter. The '* ONT' assemblies were generated in this study from the sequencing reads generated with the Nanopore MinION. Values at nodes indicate bootstrap support values. Branch lengths represent the number of substitutions per site

which produced a continuous supply of glucose from hydrolysis of the more complex wort sugars.

To ensure that the growth of the WY3711_D1 deletion strain was not impaired from the transformation process, we also compared the growth of WY3711 and WY3711_D1 on YP-Glucose and YP-Maltose in microplate cultivations and saw no significant differences in growth (Supplementary Fig. S3). Interestingly, despite showing differential maltotriose use in wort fermentations, we also did not observe any significant differences in growth when WY3711 and 

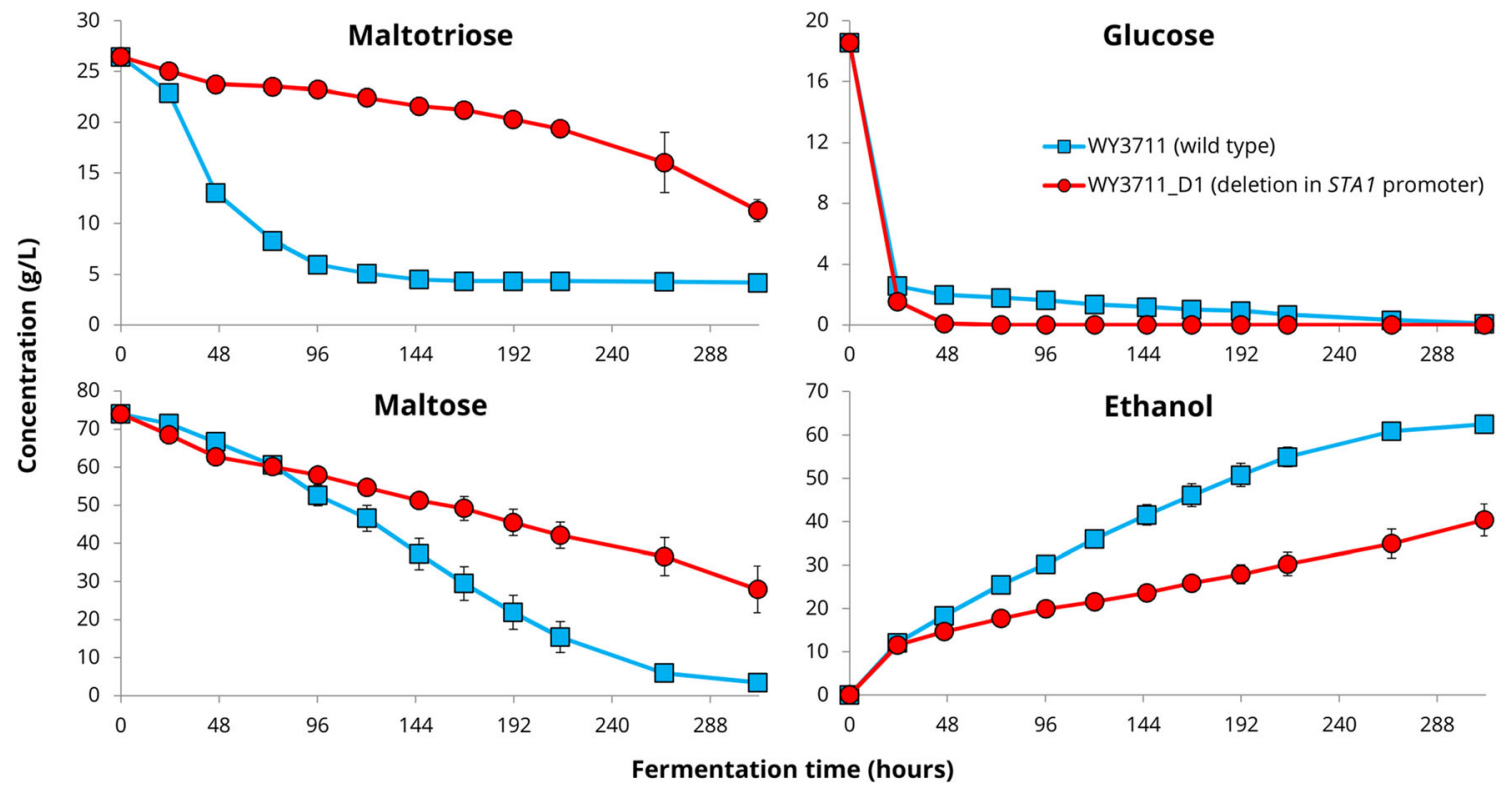

6 Decreased wort fermentation after the 1162-bp deletion in the STA1 promoter. The concentrations of fermentable wort sugars and ethanol during fermentations of $S$. cerevisiae WY3711 (blue squares)

and WY3711 D1 (1162 bp deletion in STA1 promoter; red circles) in $15^{\circ} \mathrm{P}$ wort. Error bars where visible depict the standard deviation of three replicates

WY3711_D1 were grown on YNB-Maltotriose in microplate cultivations (Supplementary Fig. S3). This would suggest that STA1 is not the only mechanism enabling maltotriose use in WY3711.

Next we deleted the STA1 open reading frame from three of the highly diastatic strains: TUM BI PA 109, TUM 71 and WY3711. The sta1 $\Delta$ deletion strains (*_S1 in Table 1) were compared to the wild-type strains in $15^{\circ} \mathrm{P}$ wort fermentations. As during the comparative fermentations of WY3711 and WY3711_D1, we observed significantly slower fermentation with the stal $\Delta$ deletion strains compared to the wild-type strains after $24 \mathrm{~h}$ of fermentation (Fig. 7a-c). The fermentations with the deletion strains lacking STA1 also appeared to finish at a lower degree of fermentation than the wild-type strains. Maltotriose use during fermentations was significantly decreased $(p<0.001$ at the end-point for all strains as determined by two-tailed Student's $t$ test) in the strains lacking STA1 compared to the wild-types (Fig. 7d-f). The wild-types of TUM PI BA 109 and TUM 71, for example, had consumed over $80 \%$ of the available maltotriose when fermentations were ended, while the stal $\Delta$ deletion strains had consumed around 5\%. Therefore, it appears as if STA1 enables efficient consumption of maltotriose from wort and is the main mechanism for maltotriose consumption in these three STA1+ strains. Interestingly, maltose use was also impaired in both WY3711_D1 (Fig. 6) and WY3711_S1 (Fig. 7f), suggesting STA1 also facilitates maltose use in some STA1+ strains. Nevertheless, the sta1 $\Delta$ deletion strains still consumed minor amounts of maltotriose, confirming that STA1 is not the sole mechanism for maltotriose use in these strains. To confirm this we measured the zero-trans uptake rate of maltotriose in these strains using $\left[\mathrm{U}_{-}{ }^{14} \mathrm{C}\right]$-maltotriose. The assay measures the change in radioactivity after incubating yeast cells with radio-labelled sugar for $1 \mathrm{~min}$ (Lucero et al. 1997). The presence of extracellular glucoamylases should not affect the zerotrans uptake rate, as yeast cells are washed twice prior to the 1min exposure to the radio-labelled sugar. Maltotriose uptake ability was detected in all three strains (Fig. 7g). As expected, the deletion of STA1 did not affect the zero-trans maltotriose uptake rate.

We queried the genome assembly of S. cerevisiae WY3711 for common maltose and maltotriose transporters (AGT1/ MAL11, MAL31 and MTT1). MTT1, a maltotriose transporter with $>90 \%$ sequence similarity to MAL31 (Dietvorst et al. 2005), appeared to be present in four copies (>95\% sequence identity; Supplementary Table S7) and likely explains the maltotriose uptake ability in WY3711. Despite this, the sta1 $\Delta$ deletion strain WY3711_S1 consumed maltotriose relatively poorly during wort fermentation. The other known maltotriose transporter, $A G T 1 / M A L 11$, appeared to be lacking from WY3711 completely, as we were unable to detect it in the genome assembly of WY3711 (Supplementary Table S7) nor did any sequencing reads align to MAL11 or MAL13 in the S288C reference genome (Supplementary Fig. S4).

We also compared the maltotriose use ability of the nineteen STA1+ strains (all belonging to the 'Beer 2' population) described in Gallone et al. (2016; data was obtained from Supplementary Table S5). We observed that the strains carrying the $1162 \mathrm{bp}$ deletion in the STA1 promoter, and therefore presumably also exhibiting lower STA1 expression, had a significantly lower ability to use maltotriose (Supplementary Fig. $\mathrm{S} 5 ; p=0.045$, Mann-Whitney $U$ test). This suggests that STA 1 

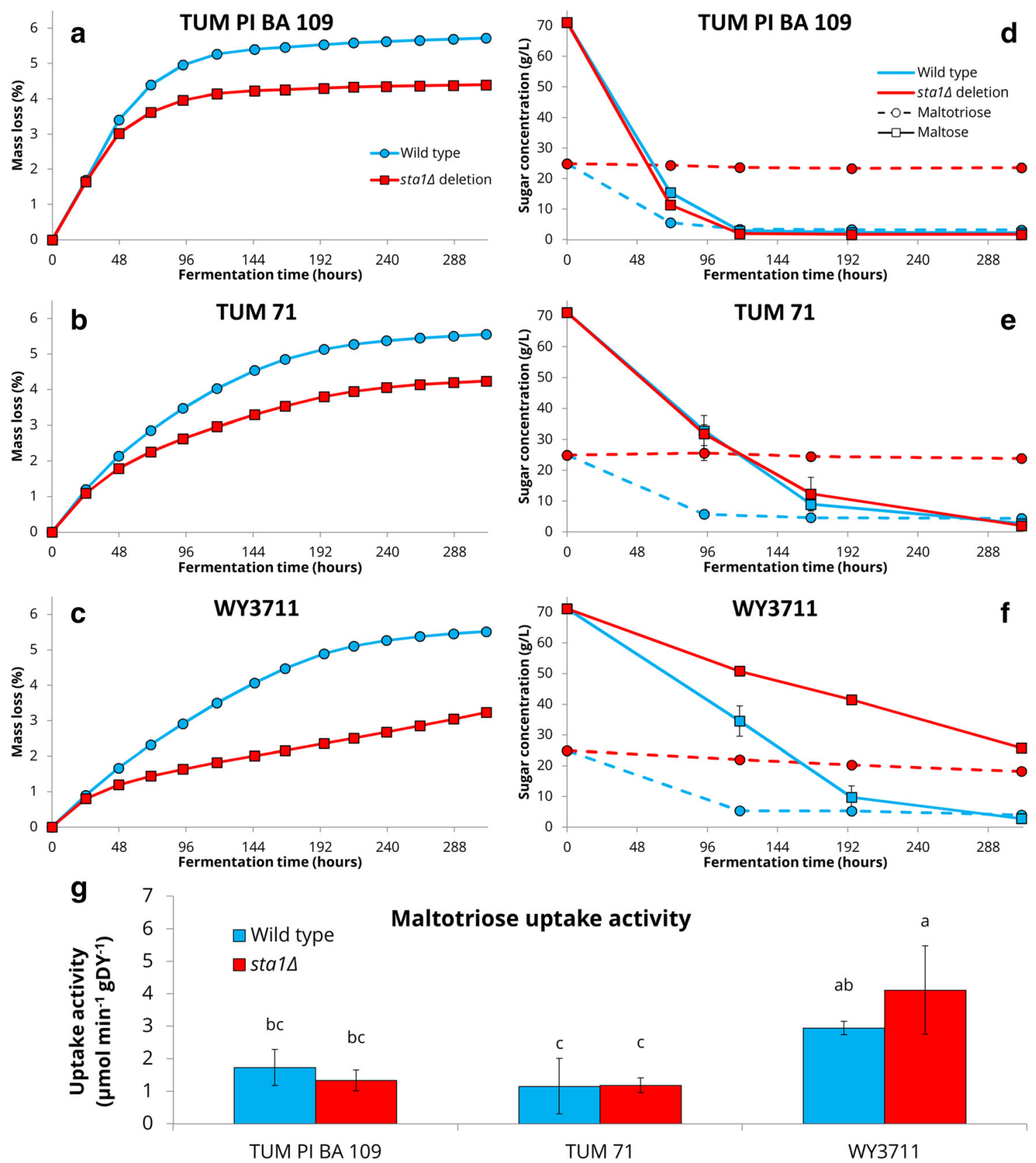

Maltotriose uptake activity

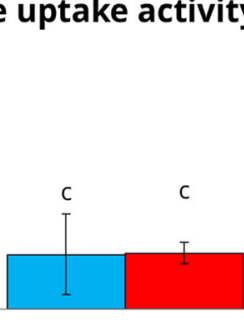

TUM 71

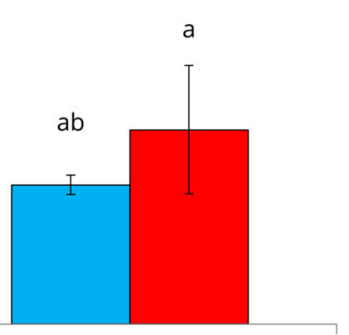

WY3711

Fig. 7 Decreased wort fermentation and maltotriose consumption after deletion of the STA1 open reading frame in three STA1+ strains. a-c The amount of mass lost as $\mathrm{CO}_{2}(\%)$ during fermentation. d-f The concentrations $(\mathrm{g} / \mathrm{L})$ of maltose (solid line, squares) and maltotriose (dashed line, circles) in the wort during fermentation. $g$ The zero-trans

has a central role in enabling maltotriose use in strains of the 'Beer 2' ('Mosaic Beer') population. However, some strains (e.g. BE034, BE083, and BE084) showed good maltotriose use despite having the deletion in the STA 1 promoter, suggesting that other mechanisms for maltotriose use exist in these strains. This was also confirmed by the fact that all three of the STA1+ strains that were tested in this study, showed zero-trans uptake rates for maltotriose.

maltotriose uptake ability $\left(\mu \mathrm{mol} \mathrm{min}^{-1}(\mathrm{~g} \text { dry yeast })^{-1}\right)$ of the strains. Values with different letters $(\mathbf{a}-\mathbf{c})$ above the bars different significantly ( $p<0.05$ by one-way ANOVA and Tukey's HSD test) (a-g) Wild-type strains in blue, stal $\Delta$ deletion strains in red. Error bars where visible depict the standard deviation of three replicates

\section{Discussion}

In this study, we aimed to elucidate genetic determinants behind the variable diastatic ability that has been observed in S. cerevisiae strains carrying the STA1 gene (i.e. strains previously known as $S$. diastaticus). While no nonsense or frameshift mutations were observed in the STAl open reading frames of strains that were screened here, we show that 
multiple STA1+ strains have a 1162-bp deletion in the STA1 promoter. The strains with the deletion showed significantly less growth in beer and on starch and consumed less dextrin when grown in media with dextrin as the sole carbon source. Reverse engineering of the most active strain confirmed the role of this deletion in decreasing the diastatic ability.

Diastatic S. cerevisiae remains a widespread and important contaminant of beer, particularly in smaller breweries where beers are seldom pasteurized, quality control is less stringent, and experimentation with different yeast strains is more common (Meier-Dörnberg et al. 2018). Using the results of this study, we developed new PCR primers which can differentiate between highly active spoilage strains harbouring the full STA1 promoter, and less active strains with the deletion in the promoter. This improves the reliability of the detection methods and can potentially reduce waste and unnecessary costs related to product recalls caused by false positives. Differentiation between highly active and benign diastatic $S$. cerevisiae has currently only been possible with timeconsuming plate-based microbiological methods (van der Aa 1998).

The variable diastatic ability in STA1+ strains appears to be a result of variable gene expression. Genomic studies have shown that mutations in non-coding regions play a large role in determining phenotype diversity by affecting gene regulation (Connelly et al. 2013; Almeida et al. 2017). Here, the deleted region contains an upstream activation sequence and transcription factor (Ste12 and Tec1) binding site (Kim et al. 2004a, b). This upstream activation sequence has been shown to facilitate gene expression (Kim et al. 2004a, b). We saw over 100-fold higher expression of STA 1 in a strain containing the full promoter, compared to two strains lacking the 1162-bp region in the promoter (natural and CRISPR/Cas9-mediated deletion), suggesting that regulation of STA1 is the cause of the differences in diastatic ability that were observed between the strains with the full and partial STA1 promoter. The results observed here highlight the importance of intergenic polymorphisms and their potential effect on phenotype. Such mutations are commonly overlooked, for example in adaptive evolution studies (Wallace-Salinas et al. 2015; Quarterman et al. 2016; Krogerus et al. 2018), and warrant more emphasis in future studies.

Wort fermentations with the engineered strains revealed that the STA1-encoded glucoamylase appears to have a central role in enabling the use of oligomeric wort sugars, such as maltotriose, by STA1+ strains during wort fermentation. It has previously been assumed that transport of maltotriose into the yeast cells is required for it to be consumed during wort fermentations (Day et al. 2002; Rautio and Londesborough 2003; Alves et al. 2008), but our results suggest that extracellular hydrolysis of maltotriose through STA1 allows for efficient consumption of maltotriose from the wort throughout fermentation. A recent study also reported extracellular hydrolysis of maltotriose by an $\alpha$-glucosidase encoded by IMA5 (Alves et al. 2018); however, in that case, maltotriose hydrolysis was only observed after an extensive lag phase. In brewing yeast where maltotriose use is enabled through the AGT1-encoded transporter, maltotriose is typically consumed from the wort only towards the later parts of fermentation when maltose concentrations are low (Rautio and Londesborough 2003). Here, in the STA1+ strains, maltotriose was consumed rapidly from the wort alongside maltose. In contrast to typical wort fermentations, where the yeast rapidly consumes the glucose from the wort, we also observed low concentrations of glucose from the extracellular hydrolysis throughout most of the active fermentation with the STA1+ strains with the full promoter. This continuous supply of glucose to the yeast presumably influences the yeast transcriptome, particularly those genes which are repressed by glucose (e.g. maltose permeases; Day et al. 2002), and this could be an interesting topic for future studies.

Brewers have traditionally associated diastatic contaminants with 'wild' yeast (Meier-Dörnberg et al. 2018), but here we show that this trait is associated with two domesticated S. cerevisiae populations, 'Beer 2'/'Mosaic beer' and 'French Guiana human' (Gallone et al. 2016; Peter et al. 2018). Strains of both the 'Beer 1'/'Ale beer' and 'Beer 2'/ 'Mosaic beer' populations have been shown to utilize maltotriose efficiently, and this trait is considered a domestication signature of brewing strains (Gallone et al. 2016). Maltotriose use in the 'Beer 1' strains has been linked to the presence and increased copy number of AGT1/MAL11, but this allele is either absent or non-functional in the 'Beer 2\% 'Mosaic beer' strains (Gallone et al. 2016). Our results suggest that STA1 is, until now, the unknown mechanism that enables efficient maltotriose use in the 'Beer 2'/'Mosaic beer' strains. We propose that the formation and retention of STA1, through the chimerization of FLOI1 and SGAI (Yamashita et al. 1987; Lo and Dranginis 1996), is an alternative evolutionary strategy for efficient utilization of sugars present in brewer's wort. The presence of STAl is expected to provide a fitness advantage to strains lacking the ability to use maltotriose in a wort environment, and this should be tested in future studies. While MTT1 also appears to be present in many 'Beer 2'/'Mosaic beer' strains, the results here suggest that extracellular hydrolysis of maltotriose seems to be the dominant route for utilization. It may be speculated that this trait was later counterselected by brewers, mediated by the deletion in the promoter. This counter-selection may have been driven by the desired specifications of the beer (e.g. not overly dry) or the need to store beer for extended periods without excessive build-up of pressure in vessels. Interestingly, chimerization of two SeMALT genes to form a functional maltotriose transporter was recently also demonstrated in two adaptive evolution studies on maltotriose fermentation (Baker and Hittinger 2019; Brouwers et al. 2019). 
The prevalence of STA1 in the 'French Guiana human' population (Peter et al. 2018) was unexpected. These isolates were obtained from various origins including fruits, animals, but mainly human faeces (Angebault et al. 2013; Peter et al. 2018). The preparation and consumption of cachiri, a traditional starch-rich fermented beverage made from cassava (La Barre 1938; Carrizales et al. 1986), is widespread among the humans that were sampled (Angebault et al. 2013), and it is possible that STA1 also provides a fitness advantage in the starch-rich environment of cachiri. The possible link between the 'Beer 2'/ 'Mosaic beer' and 'French Guiana human' strains remains unclear, and this should be clarified in future studies. The sequence similarity of the STA1 gene from S. cerevisiae WY3711 ('Beer 2') and OS899 ('French Guiana human') around the FLO11/ SGA1 junction and in the promoter suggests that they might have a common origin (Supplementary Fig. S6). In addition, because all strains of these two lineages do not contain STA1, future studies, with the aid of long-read sequencing, could also elucidate if STA 1 was acquired independently in strains from these groups, acquired by admixture, or whether STA1 was acquired by a common ancestor and later lost in some strains. The long-read assemblies that were generated here suggest that the STA1 genes are located in subtelomeric regions. As these regions are unstable and prone to recombination (Brown et al. 2010), it is possible that the 'Beer 2' strains lacking STA1 have lost it.

In conclusion, we show here that the variable diastatic ability that has been observed in STA1+ strains is a result of a deletion in the STA1 promoter, and that STA 1 also plays a central role in enabling maltotriose use during wort fermentations. This allows for the improved reliability of molecular detection methods for diastatic contaminants in beer and can be exploited for strain development where maltotriose use is desired. To further clarify the role of STA1 as a mechanism enabling maltotriose use in brewing strains, its effect on fitness in a brewing environment should be elucidated. In addition, the ability of STA1 to enable maltotriose use could be confirmed by expressing STA1 in a strain lacking transmembrane maltotriose uptake ability.

Acknowledgements We thank Mathias Hutzler and Richard Preiss for providing strains.

Author contributions $\mathrm{KK}$ conceived the study. $\mathrm{KK}, \mathrm{JK}$, and $\mathrm{BG}$ designed the experiments. KK, FM and JK conducted the experiments described in this study. KK analysed all data. KK wrote the manuscript. BG, FM and JK edited the manuscript. All authors read and approved the final manuscript.

Funding information Open access funding provided by Technical Research Centre of Finland (VTT). This work was financially supported by the Alfred Kordelin Foundation, Svenska Kulturfonden - The Swedish Cultural Foundation in Finland, Suomen Kulttuurirahasto, and the Academy of Finland (Academy Project 276480).
Data availability The datasets generated for this study can be found in the NCBI's Short Read Archive under BioProject PRJNA544899 in the NCBI BioProject database (https://www.ncbi.nlm.nih.gov/bioproject/).

\section{Compliance with ethical standards}

Conflict of interest The authors BG, JK, and FM were employed by VTT Technical Research Centre of Finland Ltd. Author KK was affiliated with VTT Technical Research Centre of Finland Ltd. The funders had no role in study design, data collection and analysis, decision to publish, or preparation of the manuscript. A provisional patent application entitled 'A method for determining a yeast having extracellular glucoamylase STA1p activity in a sample and tools and uses related thereto' (20195454) has been filed by VTT Technical Research Centre of Finland Ltd. based on the results.

Ethical approval This article does not contain any studies with human participants or animals by any of the authors.

Open Access This article is distributed under the terms of the Creative Commons Attribution 4.0 International License (http:// creativecommons.org/licenses/by/4.0/), which permits unrestricted use, distribution, and reproduction in any medium, provided you give appropriate credit to the original author(s) and the source, provide a link to the Creative Commons license, and indicate if changes were made.

\section{References}

Adam AC, Latorre-García L, Polaina J (2004) Structural analysis of glucoamylase encoded by the STA1 gene of Saccharomyces cerevisiae (var. diastaticus). Yeast 21:379-388. https://doi.org/10. 1002/yea. 1102

Almeida P, Barbosa R, Bensasson D, Gonçalves P, Sampaio JP (2017) Adaptive divergence in wine yeasts and their wild relatives suggests a prominent role for introgressions and rapid evolution at noncoding sites. Mol Ecol 26:2167-2182. https://doi.org/10.1111/mec.14071

Alves SL, Herberts RA, Hollatz C, Trichez D, Miletti LC, de Araujo PS, Stambuk BU (2008) Molecular analysis of maltotriose active transport and fermentation by Saccharomyces cerevisiae reveals a determinant role for the AGT1 permease. Appl Environ Microbiol 74: 1494-1501. https://doi.org/10.1128/AEM.02570-07

Alves SL, Thevelein JM, Stambuk BU (2018) Extracellular maltotriose hydrolysis by Saccharomyces cerevisiae cells lacking the AGT1 permease. Lett Appl Microbiol 67:377-383. https://doi.org/10. 1111/lam. 13048

Andrews BJ, Gilliland RB (1952) Super-attenuation of beer: a study of three organisms capable of causing abnormal attenuations. J Inst Brew 58:189-196. https://doi.org/10.1002/j.2050-0416.1952. tb02675.x

Angebault C, Djossou F, Abélanet S, Permal E, Ben Soltana M, Diancourt L, Bouchier C, Woerther P-L, Catzeflis F, Andremont A, D'Enfert C, Bougnoux M-E (2013) Candida albicans is not always the preferential yeast colonizing humans: a study in Wayampi Amerindians. J Infect Dis 208:1705-1716. https://doi. org/10.1093/infdis/jit389

Baker EP, Hittinger CT (2019) Evolution of a novel chimeric maltotriose transporter in Saccharomyces eubayanus from parent proteins 
unable to perform this function. PLOS Genet 15:e1007786. https:// doi.org/10.1371/journal.pgen.1007786

Bolger AM, Lohse M, Usadel B (2014) Trimmomatic: a flexible trimmer for Illumina sequence data. Bioinformatics 30:2114-2120. https:// doi.org/10.1093/bioinformatics/btu170

Brandl A (2006) Entwicklung und Optimierung von PCR-Methoden zur Detektion und Identifizierung von brauereirelevanten Mikroorganismen zur Routine-Anwendung in Brauereien. Technische Universität München, München, Germany

Brouwers N, Gorter de Vries AR, van den Broek M, Weening SM, Elink Schuurman TD, Kuijpers NGA, Pronk JT, Daran J-MG (2019) In vivo recombination of Saccharomyces eubayanus maltosetransporter genes yields a chimeric transporter that enables maltotriose fermentation. PLOS Genet 15:e1007853. https://doi. org/10.1371/journal.pgen.1007853

Brown CA, Murray AW, Verstrepen KJ (2010) Rapid expansion and functional divergence of subtelomeric gene families in yeasts. Curr Biol. 20:895-903. https://doi.org/10.1016/j.cub.2010.04.027

Carrizales V, Barrios O, González J (1986) Mejoramiento tecnológico de la producción de cachiri bebida fermentada de yuca (Manihot esculenta Crantz) de origen indígena. Acta Cient Venez 37:318-324

Cingolani P, Platts A, Wang LL, Coon M, Nguyen T, Wang L, Land SJ, Lu X, Ruden DM (2012) A program for annotating and predicting the effects of single nucleotide polymorphisms, SnpEff: SNPs in the genome of Drosophila melanogaster strain w1118; iso-2; iso-3. Fly (Austin) 6:80-92. https://doi.org/10.4161/fly.19695

Connelly CF, Skelly DA, Dunham MJ, Akey JM (2013) Population genomics and transcriptional consequences of regulatory motif variation in globally diverse Saccharomyces cerevisiae strains. Mol Biol Evol 30:1605-1613. https://doi.org/10.1093/molbev/mst073

Day RE, Rogers PJ, Dawes IW, Higgins VJ (2002) Molecular analysis of maltotriose transport and utilization by Saccharomyces cerevisiae. Appl Environ Microbiol 68:5326-5335. https://doi.org/10.1128/ AEM.68.11.5326-5335.2002

Dietvorst J, Londesborough J, Steensma HY (2005) Maltotriose utilization in lager yeast strains: MTT1 encodes a maltotriose transporter. Yeast 22:775-788. https://doi.org/10.1002/yea.1279

Erratt JA, Nasim A (1986) Allelism within the DEX and STA gene families in Saccharomyces diastaticus. MGG Mol Gen Genet 202:255256. https://doi.org/10.1007/BF00331646

Erratt JA, Stewart GG (1981) Genetic and biochemical studies on glucoamylase from Saccharomyces diastaticus. In: Stewart G, Russell I (eds) Advances in Biotechnology. Elsevier, Oxford, pp 177-183

Gagiano M, Van Dyk D, Bauer FF, Lambrechts MG, Pretorius IS (1999) Divergent regulation of the evolutionarily closely related promoters of the Saccharomyces cerevisiae STA2 and MUC1 genes. J Bacteriol 181:6497-6508

Gallone B, Steensels J, Baele G, Maere S, Verstrepen KJ, Prahl T, Soriaga L, Saels V, Herrera-Malaver B, Merlevede A, Roncoroni M, Voordeckers K, Miraglia L, Teiling C, Steffy B, Taylor M, Schwartz A, Richardson T, White C (2016) Domestication and divergence of Saccharomyces cerevisiae beer yeasts. Cell 166:13971410.e16. https://doi.org/10.1016/j.cell.2016.08.020

Gietz R, Woods RA (2002) Transformation of yeast by lithium acetate/ single-stranded carrier DNA/polyethylene glycol method. Methods Enzymol 350:87-96

Istace B, Friedrich A, D'Agata L, Faye S, Payen E, Beluche O, Caradec C, Davidas S, Cruaud C, Liti G, Lemainque A, Engelen S, Wincker P, Schacherer J, Aury J-M (2017) de novo assembly and population genomic survey of natural yeast isolates with the Oxford Nanopore MinION sequencer. Gigascience 6:1-13. https://doi.org/10.1093/ gigascience/giw018

Kim TS, Kim HY, Yoon JH, Kang HS (2004a) Recruitment of the Swi/ Snf complex by Ste12-Tec1 promotes Flo8-Mss11-mediated activation of STA1 expression. Mol Cell Biol 24:9542-9556. https://doi.org/10.1128/MCB.24.21.9542-9556.2004

Kim TS, Lee SB, Kang HS (2004b) Glucose repression of STA1 expression is mediated by the $\mathrm{Nrg} 1$ and Sfl1 repressors and the Srb8-11 complex. Mol Cell Biol 24:7695-7706. https://doi.org/10.1128/ MCB.24.17.7695-7706.2004

Kleinman MJ, Wilkinson AE, Wright IP, Evans IH, Bevan EA (1988) Purification and properties of an extracellular glucoamylase from a diastatic strain of Saccharomyces cerevisiae. Biochem J 249:163170. https://doi.org/10.1042/bj2490163

Kolmogorov M, Raney B, Paten B, Pham S (2014) Ragout-a referenceassisted assembly tool for bacterial genomes. Bioinformatics 30: i302-i309. https://doi.org/10.1093/bioinformatics/btu280

Krogerus K, Arvas M, De Chiara M, Magalhães F, Mattinen L, Oja M, Vidgren V, Yue JX, Liti G, Gibson B (2016) Ploidy influences the functional attributes of de novo lager yeast hybrids. Appl Microbiol Biotechnol 100:7203-7222. https://doi.org/10.1007/s00253-0167588-3

Krogerus K, Holmström S, Gibson B (2018) Enhanced wort fermentation with de novo lager hybrids adapted to high-ethanol environments. Appl Environ Microbiol 84:e02302-e02317. https://doi.org/10. 1128/AEM.02302-17

Kurtz S, Phillippy A, Delcher AL, Smoot M, Shumway M, Antonescu C, Salzberg SL (2004) MUMmer - Versatile and open software for comparing large genomes. Genome Biol 5:R12. https://doi.org/10. 1186/gb-2004-5-2-r12

La Barre W (1938) Native American beers. Am Anthropol 40:224-234 https://doi.org/10.1525/aa.1938.40.2.02a00040

Lambrechts MG, Pretorias IS, Sollitti P, Marmur J (1991) Primary structure and regulation of a glucoamylase-encoding gene (STA2) in Saccharomyces diastaticus. Gene 100:95-103. https://doi.org/10. 1016/0378-1119(91)90354-E

Li H (2018) Minimap2: pairwise alignment for nucleotide sequences. Bioinformatics 34:3094-3100. https://doi.org/10.1093/ bioinformatics/bty 191

Lo WS, Dranginis AM (1996) FLO11, a yeast gene related to the STA genes, encodes a novel cell surface flocculin. J Bacteriol 178:7144 7151. https://doi.org/10.1128/jb.178.24.7144-7151.1996

Lucero P, Peñalver É, Moreno E, Lagunas R (1997) Moderate concentrations of ethanol inhibit endocytosis of the yeast maltose transporter. Appl Environ Microbiol 63:3831-3836

Meier-Dörnberg T, Kory OI, Jacob F, Michel M, Hutzler M (2018) Saccharomyces cerevisiae variety diastaticus friend or foe? - spoilage potential and brewing ability of different Saccharomyces cerevisiae variety diastaticus yeast isolates by genetic, phenotypic and physiological characterization. FEMS Yeast Res. 18. https://doi. org/10.1093/femsyr/foy023

Minh BQ, Nguyen MAT, Von Haeseler A (2013) Ultrafast approximation for phylogenetic bootstrap. Mol Biol Evol 30:1188-1195. https:// doi.org/10.1093/molbev/mst024

Nguyen LT, Schmidt HA, Von Haeseler A, Minh BQ (2015) IQ-TREE: A fast and effective stochastic algorithm for estimating maximumlikelihood phylogenies. Mol Biol Evol 32:268-274. https://doi.org/ 10.1093/molbev/msu300

Pedersen BS, Quinlan AR (2018) Mosdepth: Quick coverage calculation for genomes and exomes. Bioinformatics. 34:867-868. https://doi. org/10.1093/bioinformatics/btx699

Peter J, De Chiara M, Friedrich A, Yue J-X, Pflieger D, Bergström A, Sigwalt A, Barre B, Freel K, Llored A, Cruaud C, Labadie K, Aury J-M, Istace B, Lebrigand K, Barbry P, Engelen S, Lemainque A, Wincker P, Liti G, Schacherer J (2018) Genome evolution across 1, 011 Saccharomyces cerevisiae isolates. Nature 556:339-344. https://doi.org/10.1038/s41586-018-0030-5

Pfaffl MW (2001) A new mathematical model for relative quantification in real-time RT-PCR. Nucleic Acids Res 29:45e. https://doi.org/10. 1093/nar/29.9.e45 
Pretorius IS, Modena D, Vanoni M, Englard S, Marmur J (1986) Transcriptional control of glucoamylase synthesis in vegetatively growing and sporulating Saccharomyces species. Mol Cell Biol 6: 3034-3041. https://doi.org/10.1128/MCB.6.9.3034

Quarterman J, Skerker JM, Feng X, Liu IY, Zhao H, Arkin AP, Jin Y-S (2016) Rapid and efficient galactose fermentation by engineered Saccharomyces cerevisiae. J Biotechnol 229:13-21. https://doi. org/10.1016/j.jbiotec.2016.04.041

Rantasalo A, Kuivanen J, Penttilä M, Jäntti J, Mojzita D (2018) Synthetic toolkit for complex genetic circuit engineering in Saccharomyces cerevisiae. ACS Synth Biol 7:1573-1587. https://doi.org/10.1021/ acssynbio.8b00076

Rautio J, Londesborough J (2003) Maltose transport by brewer's yeasts in brewer's wort. J Inst Brew 109:251-261. https://doi.org/10.1002/j. 2050-0416.2003.tb00166.x

Sahl JW, Lemmer D, Travis J, Schupp JM, Gillece JD, Aziz M, Driebe EM, Drees KP, Hicks ND, Williamson CHD, Hepp CM, Smith DE, Roe C, Engelthaler DM, Wagner DM, Keim P (2016) NASP: an accurate, rapid method for the identification of SNPs in WGS datasets that supports flexible input and output formats. Microb Genomics 2:e000074. https://doi.org/10.1099/mgen.0.000074

Shedlovskiy D, Shcherbik N, Pestov DG (2017) One-step hot formamide extraction of RNA from Saccharomyces cerevisiae. RNA Biol 14: 1722-1726. https://doi.org/10.1080/15476286.2017.1345417

Stemmer M, Thumberger T, del Sol KM, Wittbrodt J, Mateo JL (2015) CCTop: an intuitive, flexible and reliable CRISPR/Cas9 target prediction tool. PLoS One 10:e0124633. https://doi.org/10.1371/ journal.pone. 0124633

Tamaki H (1978) Genetic studies of ability to ferment starch in Saccharomyces: gene polymorphism. MGG Mol Gen Genet 164: 205-209. https://doi.org/10.1007/BF00267385

Teste M-A, Duquenne M, François JM, Parrou J-L (2009) Validation of reference genes for quantitative expression analysis by real-time RTPCR in Saccharomyces cerevisiae. BMC Mol Biol 10:99. https:// doi.org/10.1186/1471-2199-10-99 van der Aa Kühle A (1998) Detection and identification of wild yeasts in lager breweries. Int J Food Microbiol 43:205-213. https://oi.org/ 10.1016/S0168-1605(98)00113-5

Walker BJ, Abeel T, Shea T, Priest M, Abouelliel A, Sakthikumar S, Cuomo CA, Zeng Q, Wortman J, Young SK, Earl AM (2014) Pilon: an integrated tool for comprehensive microbial variant detection and genome assembly improvement. PLoS One 9:e112963. https://doi.org/10.1371/journal.pone.0112963

Wallace-Salinas V, Brink DP, Ahrén D, Gorwa-Grauslund MF (2015) Cell periphery-related proteins as major genomic targets behind the adaptive evolution of an industrial Saccharomyces cerevisiae strain to combined heat and hydrolysate stress. BMC Genomics 16:514. https://doi.org/10.1186/s12864-015-1737-4

Yamashita I, Hatano T, Fukui S (1984) Subunit structure of glucoamylase of Saccharomyces diastaticus. Agric Biol Chem 48:1611-1616. https://doi.org/10.1080/00021369.1984.10866345

Yamashita I, Maemura T, Hatano T, Fukui S (1985a) Polymorphic extracellular glucoamylase genes and their evolutionary origin in the yeast Saccharomyces diastaticus. J Bacteriol 161:574-582

Yamashita I, Suzuki K, Fukui S (1985b) Nucleotide sequence of the extracellular glucoamylase gene STA1 in the yeast Saccharomyces diastaticus. J Bacteriol 161:567-573

Yamashita I, Nakamura M, Fukui S (1987) Gene fusion is a possible mechanism underlying the evolution of STA1. J Bacteriol 169: 2142-2149. https://doi.org/10.1128/jb.169.5.2142-2149.1987

Yamauchi H, Yamamoto H, Shibano Y, Amaya N, Saeki T (1998) Rapid methods for detecting Saccharomyces diastaticus, a beer spoilage yeast, using the polymerase chain reaction. J Am Soc Brew Chem 56:58-63. https://doi.org/10.1094/ASBCJ-56-0058

Yue J-X, Liti G (2018) Long-read sequencing data analysis for yeasts. Nat Protoc 13:1213-1231. https://doi.org/10.1038/nprot.2018.025

Publisher's note Springer Nature remains neutral with regard to jurisdictional claims in published maps and institutional affiliations. 